\title{
Cosmological surveys with multi-object spectrographs
}

\author{
Matthew Colless \\ Research School of Astronomy and Astrophysics, Australian National University, \\ Canberra, Australia
}

\begin{abstract}
Multi-object spectroscopy has been a key technique contributing to the current era of 'precision cosmology'. From the first exploratory surveys of the large-scale structure and evolution of the universe to the current generation of superbly detailed maps spanning a wide range of redshifts, multi-object spectroscopy has been a fundamentally important tool for mapping the rich structure of the cosmic web and extracting cosmological information of increasing variety and precision. This will continue to be true for the foreseeable future, as we seek to map the evolving geometry and structure of the universe over the full extent of cosmic history in order to obtain the most precise and comprehensive measurements of cosmological parameters. Here I briefly summarize the contributions that multi-object spectroscopy has made to cosmology so far, then review the major surveys and instruments currently in play and their prospects for pushing back the cosmological frontier. Finally, I examine some of the next generation of instruments and surveys to explore how the field will develop in coming years, with a particular focus on specialised multi-object spectrographs for cosmology and the capabilities of multi-object spectrographs on the new generation of extremely large telescopes.
\end{abstract}

Keywords: cosmology, large-scale structure, multi-object spectroscopy, spectrograph, telescope

\section{INTRODUCTION}

Multi-object spectroscopy (MOS) has proved to be a richly fertile technique for probing the large-scale structure of the universe, from which it has proved possible to measure a wide range of cosmological parameters with increasing precision. For cosmological purposes, the key aspects of MOS are multiplex (the number of sources that can be simultaneously observed) and field of view (the area of sky that can be accessed at one time), since the primary requirements for cosmological surveys are the size and volume of the sample. Usually (though not invariably), the quantity being determined for each source is simply the redshift - the redward shift of spectral features resulting from the expansion of the universe during the time between emission and observation. This is (again, usually) a relatively undemanding measurement to make, requiring neither high signal-to-noise in the data nor sophistication in the analysis. Hence cosmological surveys typically reduce galaxy spectra to their redshifts and focus all their efforts on covering as large a sample as possible over as large a volume as possible.

The two key milestones in the use of MOS surveys for cosmology were, first, the step up to surveys that covered a statistically-representative volume of the relatively local universe and, second, the further step up to surveys that covered statistically-representative volumes over cosmologically significant ranges in redshift. The first step ushered in the era of 'precision cosmology' for MOS surveys, while the second step made MOS surveys a key tool for probing the nature of dark energy.

In the following sections I briefly review the past, present, and future of cosmological MOS surveys. An overview of the key cosmology surveys is provided in Figure 1, which gives a timeline of significant surveys showing the increasing multiplex and survey size resulting from the ongoing development of multi-object spectrograph systems (especially fibre MOS), and a map of the large-scale structure in the universe based on the various redshift surveys carried out with Australian Astronomical Observatory (AAO) facilities over the last 20 years.

Author email: matthew.colless@anu.edu.au

Ground-based and Airborne Instrumentation for Astronomy VI, edited by Christopher J. Evans, Luc Simard, Hideki Takami Proc. of SPIE Vol. 9908, 99081S · (c) 2016 SPIE · CCC code: 0277-786X/16/\$18 · doi: 10.1117/12.2231829 


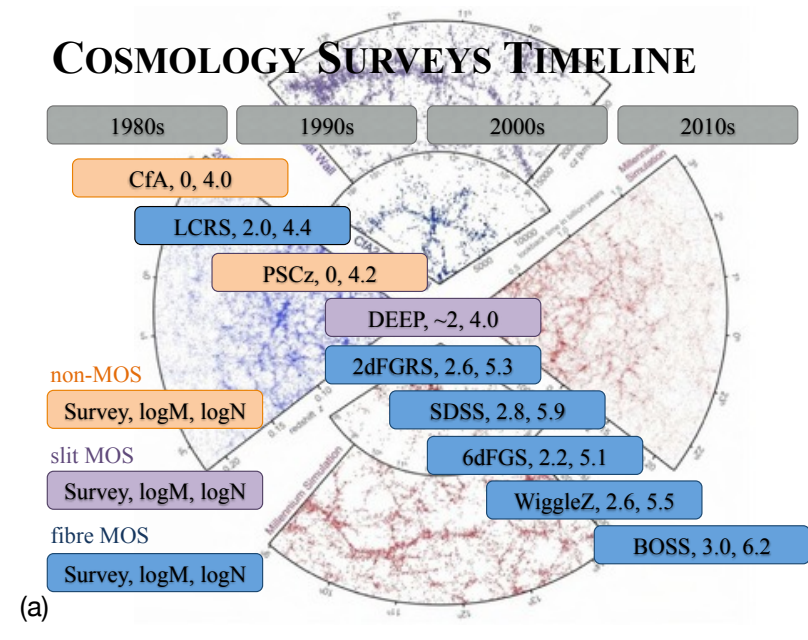

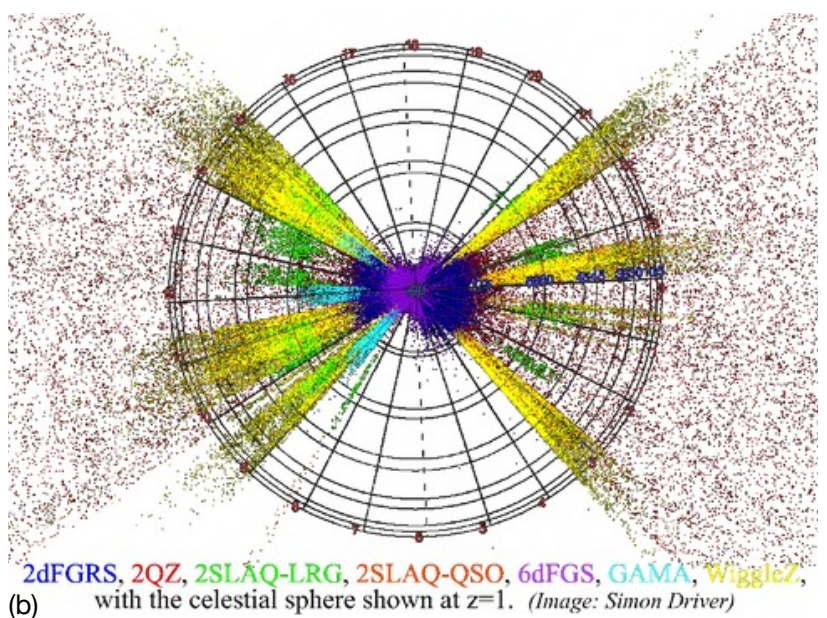

(b)

Figure 1. (a) A schematic history of cosmological redshift surveys, showing the approximatw order and dates of key surveys. The labels give the survey name and the $\log$ of the multiplex and sample size $(\log M$ and $\log N$ resp.); the colour indicates whether the survey was non-MOS (orange), slit-MOS (purple) or fibre-MOS (blue). (b) The redshift maps for the main MOS surveys carried out with AAO facilities.

\section{THE CLASSICAL PERIOD}

Due to the modest field of view and multiplex of the early MOS instruments on (mostly) 4-metre class telescopes, the initial 3applications of MOS surveys tended to be studies of relatively small volumes (often clusters of galaxies $^{1}$ ) or 'pencil-beam' surveys over a limited range of redshifts (usually looking at galaxy evolution, ${ }^{2-4}$ even though $z \ll 1$ ).

The pioneering surveys by Geller, Huchra and collaborators ${ }^{5-7}$ - which were done without the use of MOS instruments - demonstrated that there was a rich structure in the large-scale distribution of galaxies. As the theory of cosmological density perturbations ${ }^{8,9}$ was developed, it was recognized that this rich structure encoded key cosmological parameters such as the overall density of the universe, and moreover could be used to distinguish the relative contributions of ordinary 'baryonic' matter, non-relativistic 'cold' dark matter, and relativistic 'hot' dark matter. This potentiality motivated the first MOS surveys with the explicit goal of measuring cosmological cosmological parameters, in contrast to previous surveys that either sought to explore the structure of the large-scale galaxy distribution or the evolution of the galaxy population.

The most extensive of this first generation of cosmological MOS surveys were the Las Campanas Redshift Survey ${ }^{10-14}$ (LCRS), and the IRAS Point Source Catalogue Redshift Survey ${ }^{15-19}$ (PSCz). The latter was a sparse all-sky survey that did not use MOS, but the LCRS was an important forerunner to all subsequent cosmological MOS surveys. It surveyed more than 26,000 galaxies over an area of $700 \mathrm{deg}^{2}$ and reached a median depth of $z \approx 0.1$. The LCRS took 6 years on the Las Campanas 2.5-metre du Pont telescope, which had a MOS system with a multiplex of 112 and a field of view $2.1 \mathrm{deg}$ in diameter. It was an eye-opener for observational cosmologists, demonstrating the potential of MOS for probing large-scale structure and measuring the galaxy luminosity function, the correlation function and power spectrum (in 2D and 3D), the pairwise velocity distribution, and producing a catalogue of groups and clusters. However it also illuminated the size of the challenge - despite being the largest redshift survey up to that time, it was nonetheless still an order of magnitude smaller than a survey of a statistically-representative volume of the universe needed to be.

\section{THE ENLIGHTENMENT}

Two groups took on the challenge of constructing the first truly cosmological survey of the nearby universe.

One was an British and Australian team that built a revolutionary new MOS, the 2-degree Field multifibre spectrograph $(2 \mathrm{dF})$ for the 4-metre Anglo-Australian Telescope. ${ }^{20}$ As its name implies, 2dF had a 2-degree 

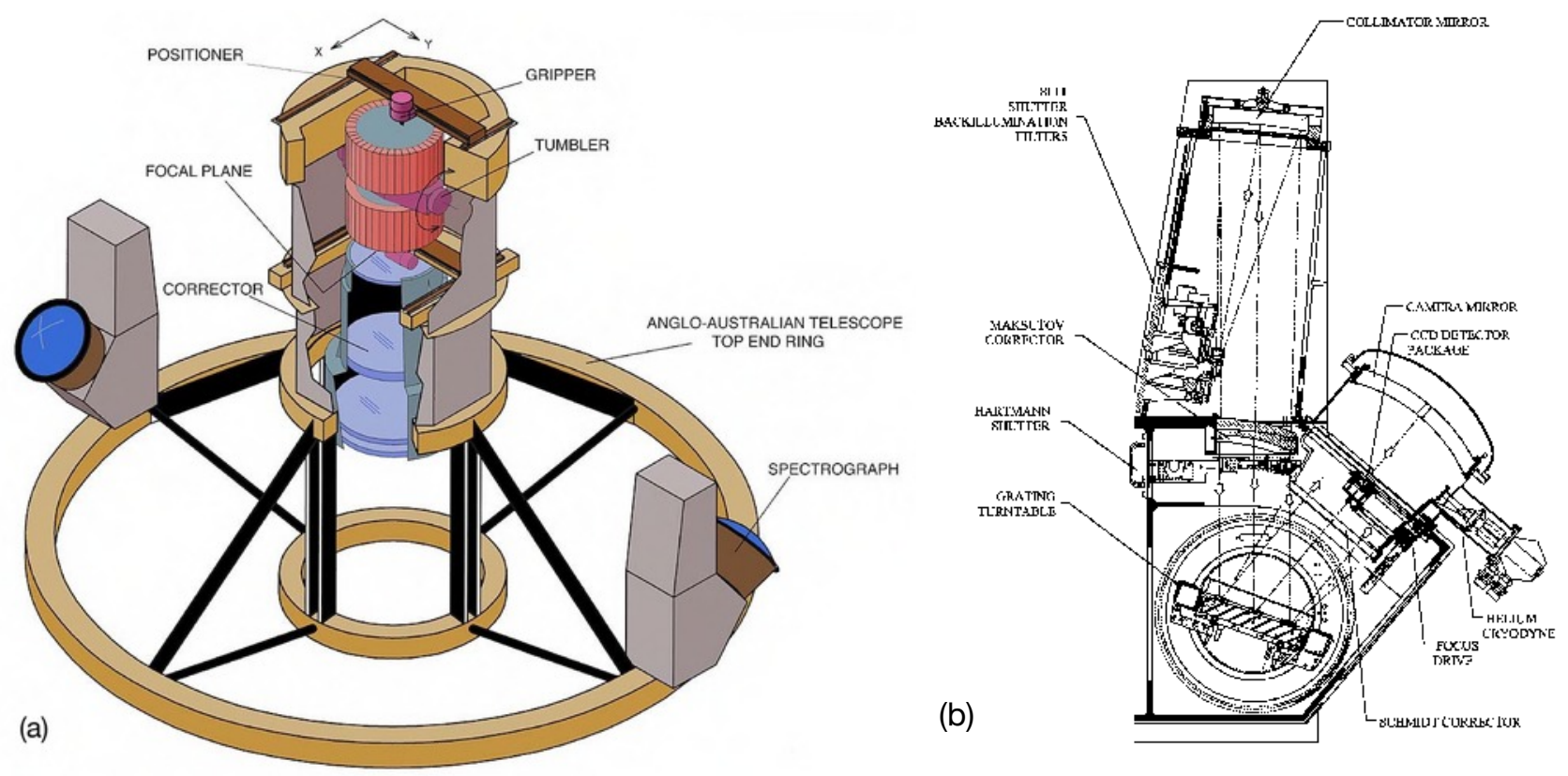

Figure 2. (a) Schematic of the 2dF topend ring for the 3.9-metre Anglo-Australian Telescope (AAT), showing the location of the two spectrographs, and a cutaway view of the corrector and positioner system. (b) A section view of one of the $2 \mathrm{dF}$ spectrographs showing the main subsystems.

diameter field of view (the largest on any on a 4-metre-class telescope), a multiplex of 400, and used a robotic fibre positioner that facilitated rapid automatic reconfiguration. ${ }^{21,22}$ The $2 \mathrm{dF}$ fibre positioning system was considered technically challenging and risky at the time it was being constructed (the early 1990s), but it proved effective and reliable in practice. ${ }^{23} 2 \mathrm{dF}$ 's wide field of view was achieved with a corrector lens incorporating an atmospheric dispersion compensator, which was an essential innovation in a system aiming to achieve wide spectral coverage with small aperture fibres. The robot positioner placed fibres sequentially at the rate of one every $6 \mathrm{~s}$ with a precision of $0.3 \operatorname{arcsec}$ (corresponding to $20 \mu \mathrm{m}$ ). Figure 2a shows the $2 \mathrm{dF}$ topend ring, with the corrector, positioner and the two spectrographs. Because it took about an hour to reconfigure a complete set of fibres, $2 \mathrm{dF}$ had a double-buffering system with two field plates each having 400 fibres. While the fibres on one field plate were being reconfigured by the robot, the second field plate was being observed; at the end of an observation the two field plates were tumbled into the other position and the process repeated. The $2.1 \operatorname{arcsec}(140 \mu \mathrm{m})$ diameter fibres fed a pair of dual-channel spectrographs that offered spectral resolving powers $(R=\lambda / \mathrm{FWHM})$ between $R=500$ and $R=2000$ covering wavelength ranges of $440 \mathrm{~nm}$ and $110 \mathrm{~nm}$ respectively. The overall throughput of the entire $2 \mathrm{dF}$ system peaked at about $5 \%$ around $600 \mathrm{~nm}$. The layout of the $2 \mathrm{dF}$ spectrographs is shown in Figure 2b.

The $2 \mathrm{dF}$ MOS system was designed from the outset to enable a massive galaxy redshift survey to test the cosmological model and measure its key parameters. Over a period of 5 years from 1997 to 2002, the 2dF Galaxy Redshift Survey (2dFGRS) measured 221,000 redshifts over $\sim 1500 \mathrm{deg}^{2}$ with a median depth of $z \sim 0.11$ (corresponding to a volume of $\sim 0.12 \mathrm{Gpc}^{3}$ ), making it the first cosmological redshift survey to capture a statistically-representative sample of the universe. ${ }^{24,25}$

The main cosmological results from 2dFGRS related to the nature of the large-scale structure, the overall density of the universe, and the nature of its massive constituents. ${ }^{26-31}$ The survey precisely determined the statistical properties of the large-scale structure of the galaxy distribution (via the galaxy power spectrum or, equivalently, the galaxy correlation function) over size scales from about $1 \mathrm{Mpc}$ to about $300 \mathrm{Mpc}$. The properties of the galaxy distribution confirmed the generally accepted paradigm that the large-scale structure grows by gravitational instability in a way that is qualitatively and quantitatively consistent with the standard model of gravitational amplification of quantum fluctuations emerging from the Big Bang. From the power 


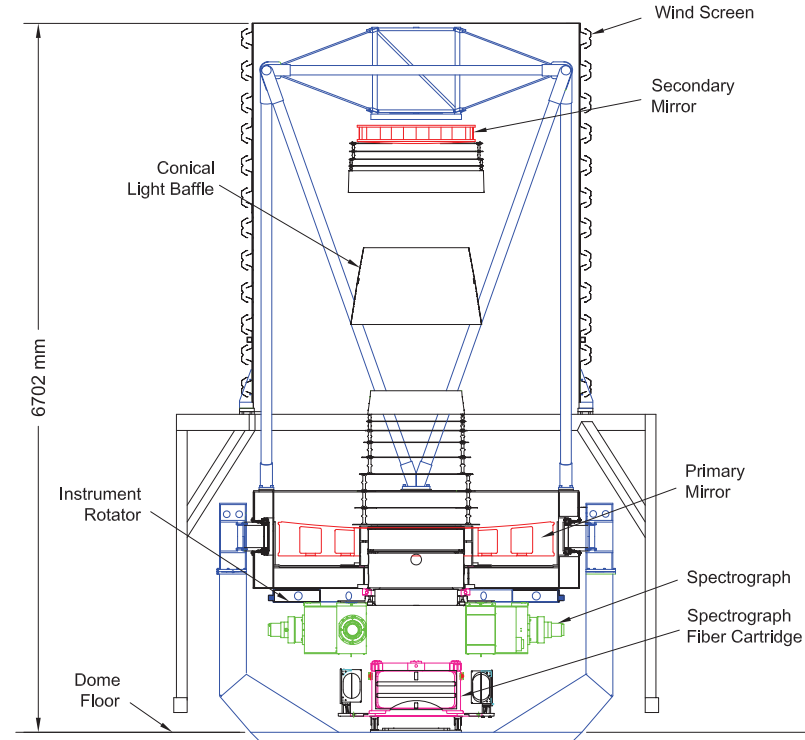

(a)

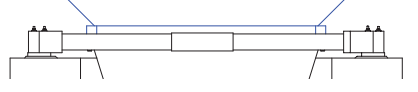

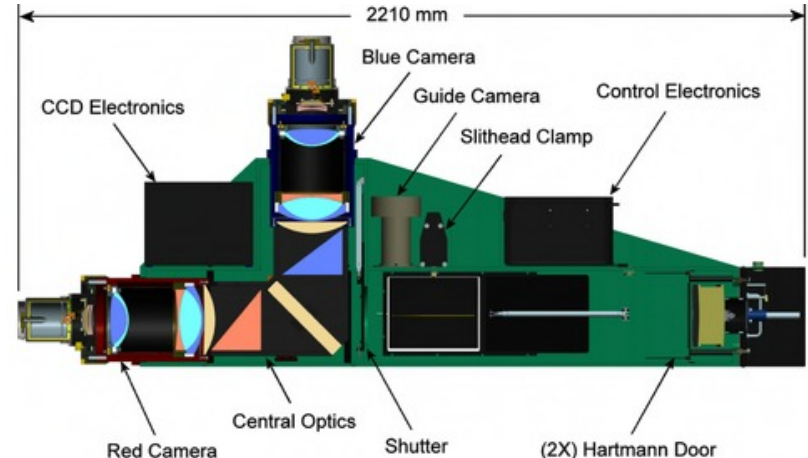

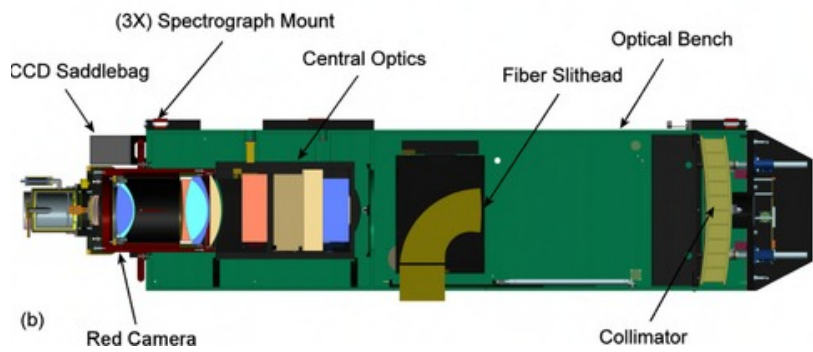

Figure 3. (a) Schematic of the Apache Point Observatory 2.5-metre telescope showing the location of the spectrographs, ${ }^{34}$ with a fiber cartridge in the retracted position. The twin spectrographs mount to the back of the Cassegrain instrument rotator adjacent to the focal plane with sufficient separation to allow installation and removal of the imaging camera and fiber cartridges. (b) Two views of the SDSS spectrographs ${ }^{34}$ and the main opto-mechanical subassemblies: the fiber slithead, the collimator, the central optics, and the red and blue channel cameras.

spectrum and redshift-space distortions, 2dFGRS obtained an estimate for the total density of all types of matter in the universe of $\Omega_{M}=0.230 .02$; the uncertainty of less than $10 \%$ on this figure was one of major steps towards precision cosmology from redshift surveys. Moreover, 2dFGRS was able to show that the fraction of the total matter density in baryons is $18 \%$, consistent with a baryon density of $\Omega_{B}=0.04$, as found from the cosmic microwave background anisotropies and Big Bang nucleosynthesis models. On the other hand, relativistic matter such as neutrinos makes up less than $13 \%$ of the overall matter density, implying an upper limit on the total mass of the three neutrino species of $0.7 \mathrm{eV}$.

Contemporaneously with the 2dFGRS, an even more ambitious project, the Sloan Digital Sky Survey (SDSS), was being carried forward by a largely US-based team. ${ }^{32,33}$ Whereas 2dFGRS relied on photographic sky surveys for its input target catalogue, the SDSS project paired a CCD imaging survey with a MOS spectral survey. SDSS used a purpose-built 2.5-metre telescope with a $3 \mathrm{deg}$ diameter field of view at Apache Point Observatory, and its MOS instrument ${ }^{34}$ had 640 fibres, each $3 \operatorname{arcsec}(180 \mu \mathrm{m})$ in diameter, that could be positioned over the $7 \mathrm{deg}^{2}$ field (see Figure 3a). Unlike 2dF's robotic system, the SDSS fibre system was a plug-plate design requiring manual positioning of the fibres. The twin SDSS spectrographs (see Figure 3b) were each fed by 320 fibres, and utilized a simple optical layout with reflective collimators, gratings, all-refractive cameras, and state-of-the-art CCD detectors to record the spectra from these fibres simultaneously in two channels over the wavelength range from $390 \mathrm{~nm}$ to $910 \mathrm{~nm}$ at a resolving power $R \approx 2000$. The overall efficiency of the spectrographs peaked at about $17 \%$ in the blue channel and $22 \%$ in the red channel.

The original SDSS survey ${ }^{35}$ (comprising SDSS-I and SDSS-II) ran for 9 years from 2000 to 2008. It imaged an area of 11,663 $\mathrm{deg}^{2}$ and obtained galaxy spectra over $8032 \mathrm{deg}^{2}$, measuring redshifts for 930,000 galaxies with a median redshift of $z \approx 0.1$ (corresponding to $\sim 0.5 \mathrm{Gpc}^{3}$ ). The cosmological results from SDSS $^{36-39}$ covered similar ground to those of 2dFGRS, but ultimately achieved higher precision due to both the larger size of the SDSS sample and the better quality of the CCD imaging and photometry.

With the 2dFGRS and SDSS surveys, MOS spectroscopy had made observations of large-scale structure a tool 
Table 1. A list of the telescope:instrument combinations used to perform various MOS surveys (cosmological and other).

\begin{tabular}{|c|c|}
\hline Telescope $:$ Instrument & Survey(s) \\
\hline AAT : AA $\Omega$, HERMES, SAMI & WiggleZ, GAMA, GALAH, SAMI \\
\hline UKST : 6dF, TAIPAN & RAVE, 6dFGS, Taipan \\
\hline SDSS $2.5 \mathrm{~m}:$ SDSS, BOSS, MaNGA & SDSS, BOSS, eBOSS, MaNGA \\
\hline WHT $:$ ISIS, WEAVE & WEAVE \\
\hline GTC : OSIRIS, EMIR, MEGARA & \\
\hline VLT $:$ VIMOS, KMOS, MUSE, & VANDELS, VIPERS, LEGA-C,... \\
\hline MOONS & 4MOST, WAVES \\
\hline VISTA : 4MOST & LEGUE \\
\hline LAMOST & CALIFA \\
\hline Calar Alto : CALIFA & PRIMUS \\
\hline Magellan : PRIMUS & FastSound \\
\hline Subaru : FMOS, PFS & HETDEX \\
\hline HET : VIRUS & GAIA \\
GAIA & DESI \\
\hline KPNO 4m : DESI & Euclid \\
\hline Euclid & $\ldots$ \\
\hline LSST:MOS / MSE / WFIRST & \\
\hline
\end{tabular}

for 'precision cosmology' - though that initial level of 'precision' seems rather imprecise by today's standards. One of the key outcomes from these surveys was the detection of the baryon acoustic oscillation (BAO) signature in the galaxy distribution. ${ }^{30,38,39}$ The BAO 'standard ruler' has been a key tool in subsequent cosmological surveys seeking to probe the nature of dark energy, using the evolution of the expansion rate and geometry of the universe as means of determining the dark energy equation of state.

\section{THE MODERN ERA}

Following the outstanding success of the 2dFGRS and SDSS-I/II surveys there has been a procession of follow-on cosmological MOS surveys addressing a range of different issues. Table 1 lists the main recent MOS surveys (both cosmological and other) and the telescope/instrument combinations with which they have been carried out. Notable amongst the various cosmological MOS surveys in this list are the subsequent SDSS surveys (including BOSS and eBOSS), the WiggleZ survey on the Anglo-Australian Telescope, and the 6dFGS survey on the UK Schmidt Telescope.

\subsection{6dFGS}

The $6 \mathrm{dF}$ Galaxy Survey (6dFGS) is a cosmological survey of the relatively local $(z<0.1)$ universe ${ }^{40-43}$ differing from others in combining a redshift survey with a peculiar velocity survey. The redshift survey ${ }^{41,44}$ was relatively standard, except that it covered whole southern hemisphere apart more than 10 deg from the Galactic Plane (at total area of 17,000 deg) and was restricted to the brightest 125,000 galaxies in the local universe. The peculiar velocity survey ${ }^{45-47}$ used Fundamental Plane distances for early-type galaxies, in combination with their redshifts, to measure the peculiar velocities (i.e. non-Hubble-flow motions) for about 8000 galaxies at distances $c z<16,000 \mathrm{~km} \mathrm{~s}^{-1}$. Peculiar velocity surveys, by adding direct measurements of the motions of galaxies due to the effective gravitational force of the surrounding matter, provide additional, complementary information to redshift surveys regarding both the mass distribution and the nature of gravity. However, available methods for measuring galaxies' distances independent of their redshifts are not precise (e.g. the Fundamental Plane provides distances with typical errors of about 20\%), and as a result they can only measure peculiar velocities effectively at relatively small distances (i.e. relatively low redshifts).

The 6dFGS observations were carried out over 5 years from 2001 to 2006 using the 6-degree Field (6dF) MOS system on the UK Schmidt Telescope (UKST). ${ }^{41,48-51}$ The $6 \mathrm{dF}$ system had 150 science fibers, each 6.7 arcec 

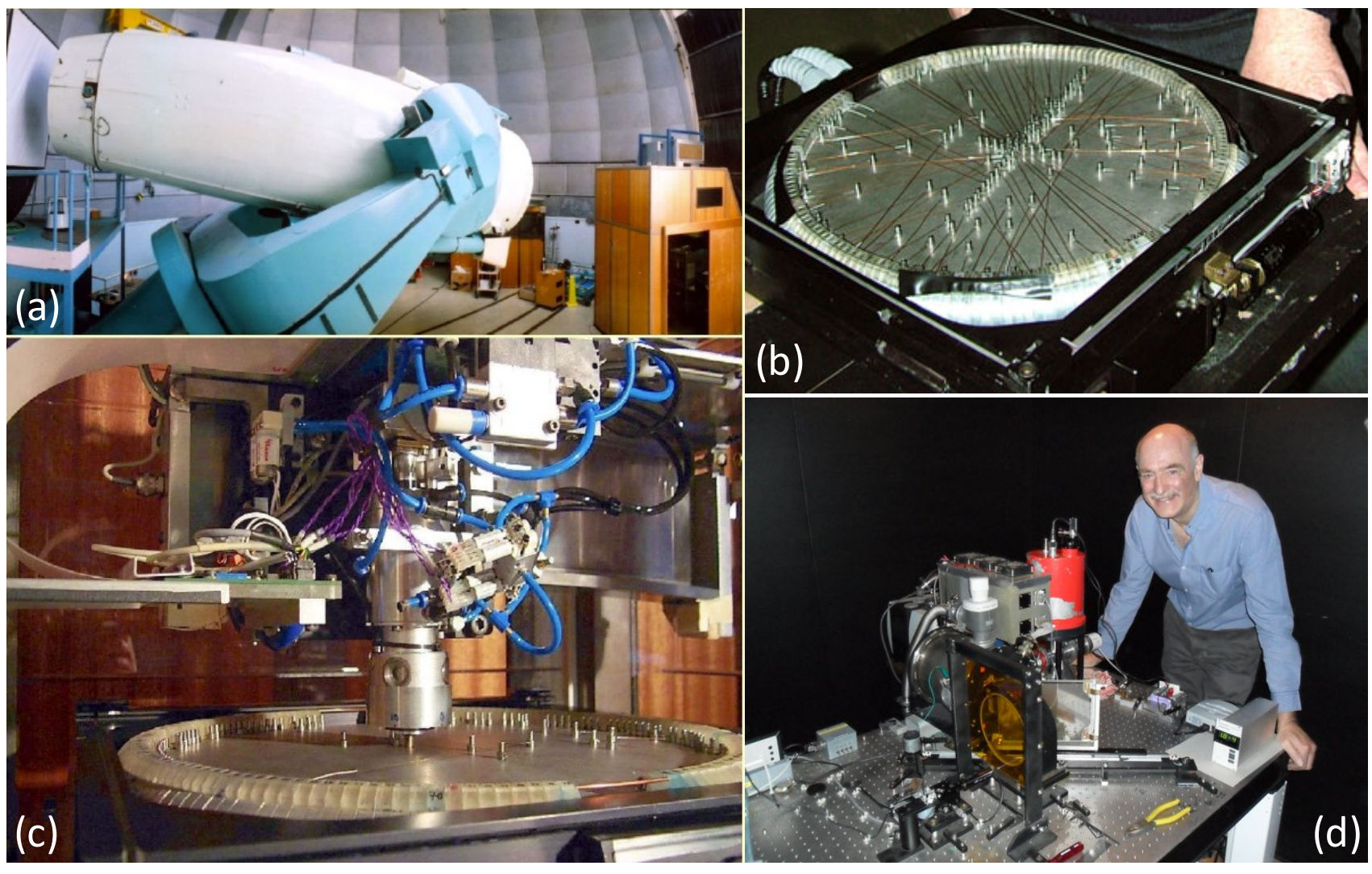

Figure 4. Images of the 6dF instrument. (a) The UK Schmidt Telescope (UKST) at Siding Spring Observatory. (b) The $6 \mathrm{dF}$ field plate holder, showing optical fibres and buttons on the convex field plate. (c) The $6 \mathrm{dF} r-\theta-z$ robot. (d) The $6 \mathrm{dF}$ spectrograph with its designer, Fred Watson.

$(100 \mu \mathrm{m})$ in diameter, that could be positioned over the 5.7-degree field of the UKST (see Figure 4a \& b). This made $6 \mathrm{dF}$ the ideal instrument for full-sky spectroscopic surveys of relatively sparse $\left(<50 \mathrm{deg}^{-2}\right)$, bright $(\mathrm{V}<17)$ objects. In terms of the $\mathrm{A} \Omega$ (telescope aperture $\times$ field of view) figure of merit, $6 \mathrm{dF}$ on the 1.2-metre UKST has about $75 \%$ of the survey power of $2 \mathrm{dF}$ on the 3.9-metre Anglo-Australian Telescope (AAT), but with the operational advantage that it was entirely given over to survey observations. Because of the curved focal plane of the UKST, $6 \mathrm{dF}$ used a 3 -axis $r-\theta-z$ robot (see Figure 4c) with a curved radial arm to position the individual fibres, which were contained in an adapted version of the Schmidt photograph plate holder. The robot was located off the telescope, and the plate holders had to be manually mounted in the UKST. The relatively large 'buttons' that magnetically held the fibres on the field plate, combined with the small platescale of the UKST, meant that targets closer than 5.7 arcmin could not be observed simultaneously. The fibres fed a floor-mounted, fixed format spectrograph (see Figure 4d), which used reflection gratings up to October 2002 and thereafter volume phase holographic gratings. The limited size of the CCD detector meant that, while all 150 fibres fitted on the detector simultaneously, full spectral coverage from $\sim 400 \mathrm{~nm}$ to $\sim 800 \mathrm{~nm}$ required two exposures with different gratings. The peak system efficiency was about $11 \%$ at wavelengths near the gratings' blaze angles.

The main cosmological results from the $6 \mathrm{dFGS}$ relate to local (i.e. $z \approx 0$ ) measurements of key parameters, which are important because they require little or no reliance on an assumed cosmological model to interpret them. Thus the 6dFGS redshift survey yielded a direct and independent estimate of the local Hubble constant ${ }^{52}\left(H_{0}\right)$ based on the standard ruler provided by the baryonic acoustic oscillations (BAO) - that agrees well with the value obtained by the Planck satellite extrapolating from the cosmic microwave background (CMB) at $z \approx 1100$ assuming the standard $\Lambda$ CDM cosmology. The 6dFGS similarly provide low-redshfit measurements of the product of the normalisation of the matter power spectrum $\left(\sigma_{8}\right)$ and the growth rate of large-scale structure ${ }^{53}$ derived 

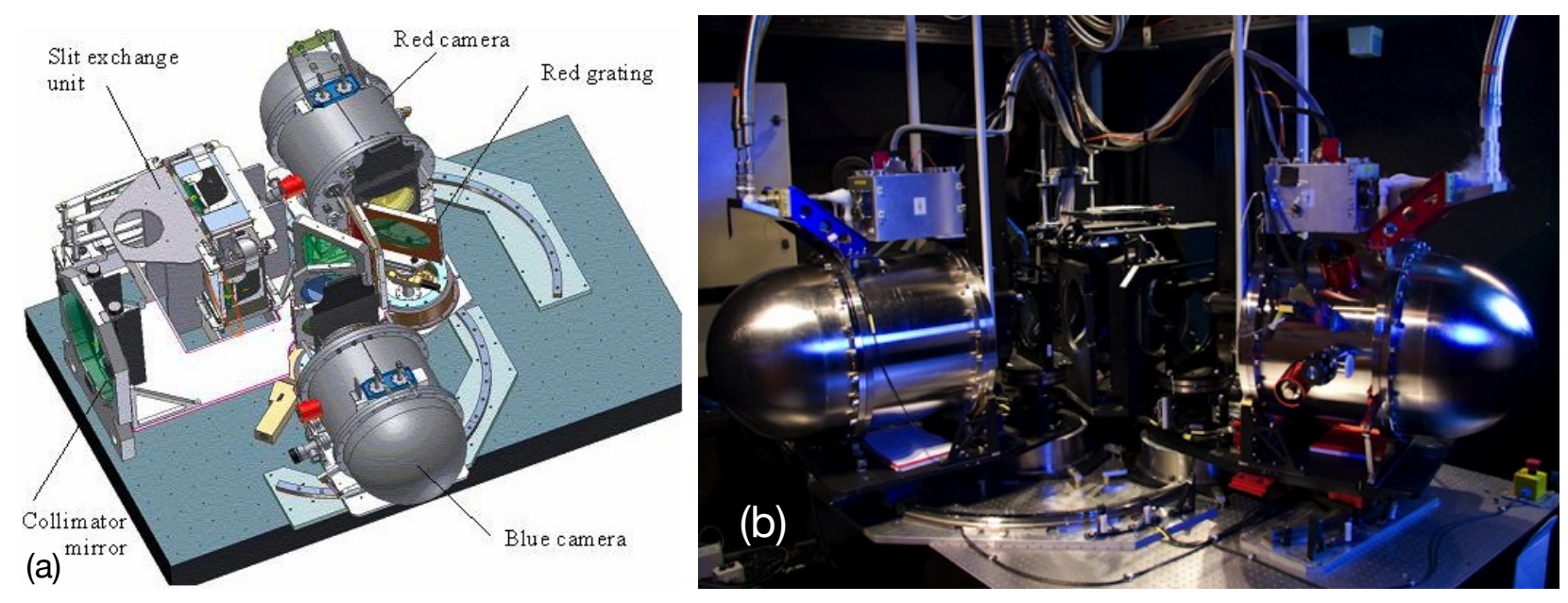

Figure 5. (a) A schematic of the AAOmega spectrograph showing the main components of the instrument. (b) A photo of the AAOmega spectrograph in operation on the AAT.

from the redshift-space distortions in the two-point galaxy correlation function. This low-redshift constraint was combined with the very high-redshift CMB constraint to confirm that the growth of structure over the history of the universe is consistent with the model for gravity provided by General Relativity. By contrast, the peculiar velocity measurements have no high-redshift counterparts, but are complementary to the redshift survey. After careful calibration of the Fundamental Plane, ${ }^{45}$ the 6dFGS was able to measure peculiar velocities for nearly 8000 early-type galaxies and map the large-scale velocity field ${ }^{47}$ in the southern hemisphere out to $\sim 16,000 \mathrm{~km} \mathrm{~s}^{-1}$. This observed velocity field was used to determine the rms bulk motions ${ }^{54}$ on scales of $50-70 h^{-1} \mathrm{Mpc}$ and to make a direct comparison with the predicted velocity field derived from the redshift survey density field. ${ }^{55}$ The 6dFGS peculiar velocities also allowed the first-ever direct measurement of the velocity power spectrum, ${ }^{56}$ which provided another means of measuring the growth rate of structure and showing that it is scale-independent up to scales of at least $300 h^{-1} \mathrm{Mpc}$.

\subsection{WiggleZ}

After the conclusion of the 2dFGRS, the original 2dF spectrographs were replaced with the AAOmega doublebeam spectrograph. ${ }^{57-59}$ AAOmega is a bench-mounted system an $\mathrm{f} / 3.15$ Schmidt collimator, a dichroic beamsplitter, volume phase holographic (VPH) gratings, and articulated f/1.3 Schmidt cameras (see Figure 5). It accommodates 392 fibers and covers the wavelength range $370 \mathrm{~nm}$ to $950 \mathrm{~nm}$ at spectral resolutions from $R=1000$ to $R=7500$. It is floor-mounted in a thermally isolated environment with a fiber cable running $38 \mathrm{~m}$ to the AAT's prime focus. Despite the long fibre run, AAOmega achieves a throughput of approximately $20 \%$ in both the blue and red arms, a gain of more than a factor of 2 over the $2 \mathrm{dF}$ spectrographs. This improvement is due to a number of factors: the use of highly efficient VPH gratings and optical coatings, and to new higher-performance CCDs. AAOmega also improved on $2 \mathrm{dF}$ by a factor of 2 in resolution, while the spectral stability is an order of magnitude better. AAOmega commenced science observations at the AAT in early 2006 and is still in high demand today, being used with the (refurbished) $2 \mathrm{dF}$ fiber positioning system, the KOALA ${ }^{60,61}$ wide-field IFU feed, and the SAMI ${ }^{62,63}$ multi-IFU system.

AAOmega has been used for a number of galaxy surveys, including the ongoing GAMA ${ }^{64}$ and SAMI ${ }^{63,65}$ surveys of galaxy properties and evolution, and the $2 \mathrm{SLAQ},{ }^{66}$ Wigglez ${ }^{67}$ and ongoing OzDES ${ }^{68}$ surveys which had cosmological goals. Of the cosmological surveys using AAOmega, WiggleZ, which focussed on the evolution of the geometry of the universe and the growth of structure at redshifts up to $z \approx 1$, has had the greatest impact.

WiggleZ ${ }^{67,69}$ measured redshifts for 238,000 galaxies starforming galaxies with $0.2 \lesssim z \lesssim 1\left(z_{\text {median }}=0.6\right)$ in 7 regions covering approximately $1000 \mathrm{deg}^{2}$ of sky with a total volume of $\sim 1 \mathrm{Gpc}^{3}$. The main results from the WiggleZ survey were: (a) using the BAO standard ruler to measure the geometry of the universe, by mapping the distance-redshift relation and measuring the cosmic expansion history by combining the Alcock-Paczynski 


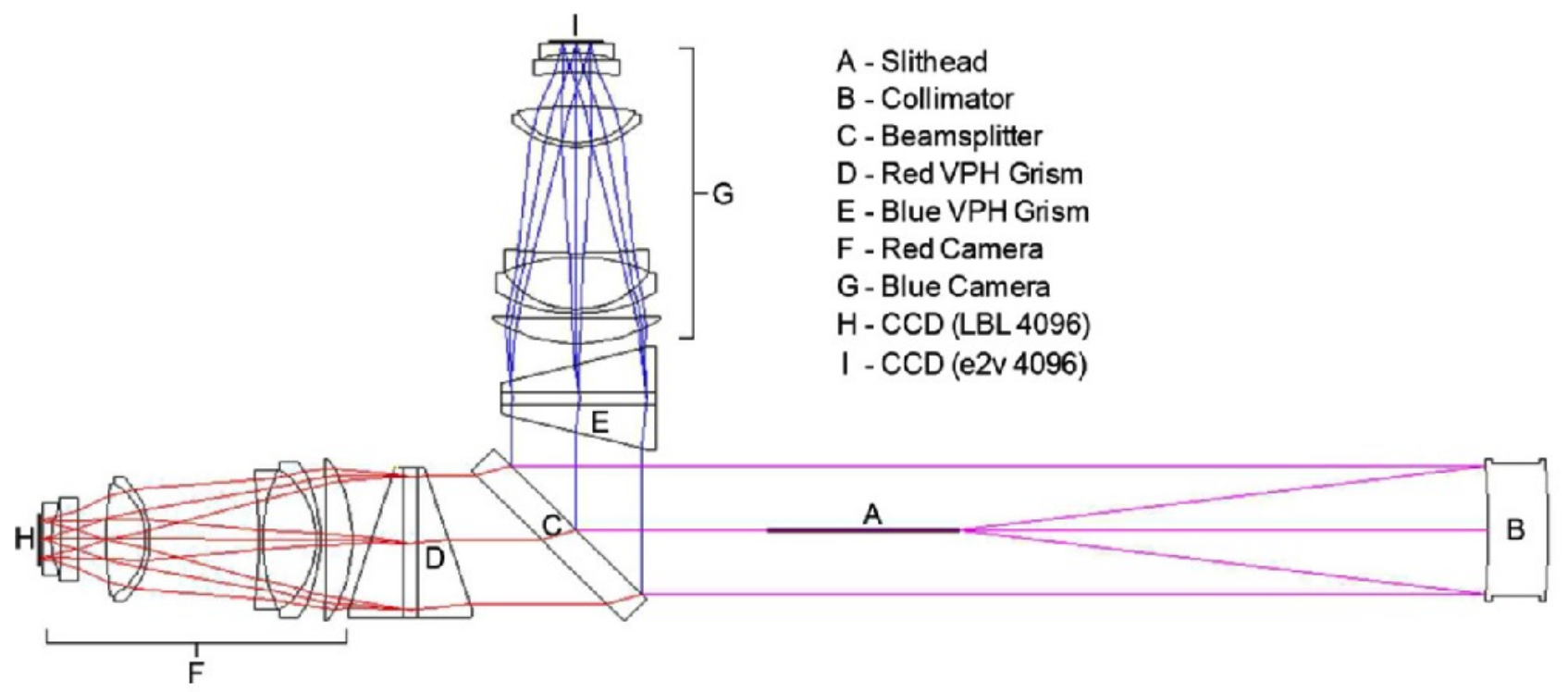

Figure 6. The optical layout for the upgraded SDSS spectrographs used for the BOSS and eBOSS surveys. ${ }^{34}$

test and distant supernovae; ${ }^{70-73}$ (b) measuring the growth rate of large-scale structure using redshift space distortions of the two- and three-point galaxy correlation functions $;{ }^{74-77}$ (c) jointly measuring the expansion and growth history out to $z \approx 1 ;^{78}$ (d) constraining the sum of the neutrino masses; ${ }^{79}$ (e) tracking the transition to large-scale cosmic homogeneity; ${ }^{80}$ and (f) fitting the galaxy power spectrum to determine six key cosmological parameters $\left(\Omega_{\mathrm{b}} h^{2}, \Omega_{\mathrm{CDM}}, H_{0}, \tau, A_{\mathrm{s}}, n_{\mathrm{s}}\right)$ and five supplementary parameters $\left(n_{\mathrm{run}}, r, w, \Omega_{\mathrm{k}}, \sum m_{\nu}\right)$, and showing that all are consistent with the standard $\Lambda \mathrm{CDM}$ model. ${ }^{69}$

\subsection{BOSS \& eBOSS}

The current state-of-the-art in precision cosmology MOS surveys is represented by the recently completed Baryon Oscillation Spectroscopic Survey ${ }^{81-84}$ (BOSS, part of SDSS-III) and the ongoing extended BOSS ${ }^{85}$ (eBOSS, part of SDSS-IV). These surveys were enabled by a 2009 upgrade to the original SDSS spectrographs ${ }^{34}$ on the Apache Point Observatory 2.5-metre telescope, just as WiggleZ and other AAT surveys were enabled by AAOmega replacing the original $2 \mathrm{dF}$ spectrographs. As with AAOmega, the upgrade to the SDSS spectrographs involved volume phase holographic gratings and more modern CCD detectors, and improved the peak efficiency by nearly a factor of 2, while extending the spectral range to $360-1000 \mathrm{~nm}$ and increasing the multiplex from 640 to 1000 fibers (500 per spectrograph). To achieve the higher multiplex, retain the spectral sampling given the smaller CCD pixels, and match the source size for the more distant BOSS galaxies, the fiber diameter was reduced from 3 arcsec to 2 arcsec. The overall layout of the upgraded spectrographs is the same as the original SDSS spectrographs (see Figure 3) but with a revised central optics assembly, as illustrated in Figure 6. As with the SAMI multi-IFU feed for AAOmega, the new SDSS spectrographs have been provided with multi-IFU capability as part of the MaNGA survey ${ }^{86}$ that is obtaining spatially resolved spectroscopy for $\sim 10,000$ galaxies.

BOSS observations were performed from 2008 to 2014, starting with imaging in 2008 and spectroscopy from 2009, when the upgraded SDSS spectrographs became available. The final data release ${ }^{84}$ (SDSS DR12) was made public in 2015, and contained spectra for 1.37 million unique galaxies and QSOs, made up of 862,735 galaxies from the LOWZ target catalogue $(0.15<z<0.4)$, 343,160 galaxies from the CMASS target catalogue $(0.4<z<0.8)$, and 158,917 QSOs with $2.15<z<3.5$ used to study large-scale structure in the Lyman-alpha forest. The BOSS spectroscopy covers an effective area of $9376 \mathrm{deg}^{2}$ over two large contiguous regions in the north and south Galactic caps.

Some of the main cosmological results of the BOSS survey, and relevant preceding surveys, are summarized ${ }^{85}$ in Figure 7. The lefthand panel shows predictions and observations of the relation between comoving distance and 

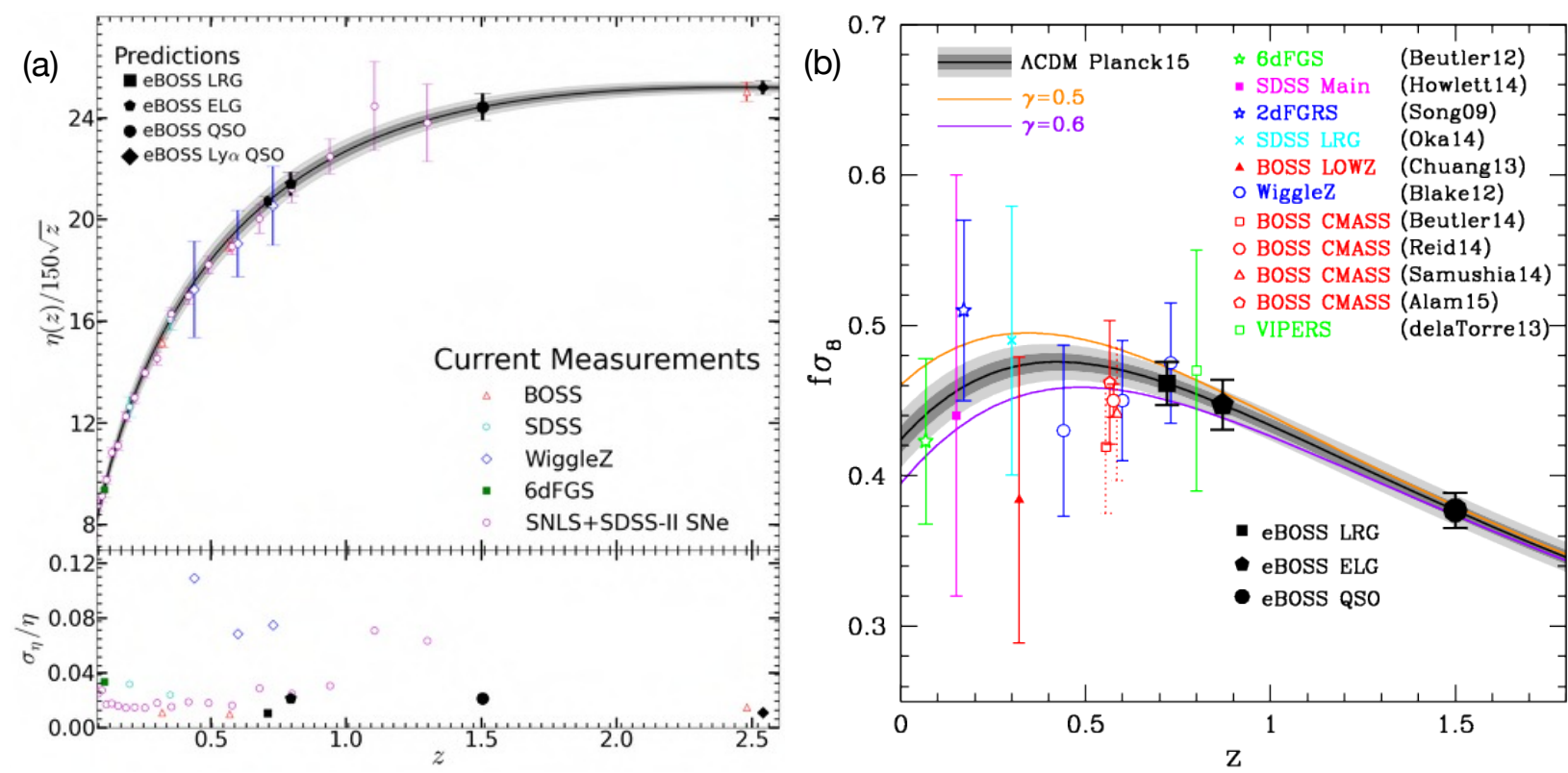

Figure 7. Measurements of the distance-redshift relation and the growth rate of structure from MOS surveys. ${ }^{85}$ (a) The comoving distance $\eta(z)$ versus redshfit $z$ relation predicted from the Planck $\Lambda$ CDM model (shown as the solid black curve, with $1 \sigma$ and $2 \sigma$ uncertainties in gray); the existing measurements from BAO surveys (6dFGS, SDSS, WiggleZ and BOSS); the expected measurements from eBOSS; and a compilation of SNe Ia measurements. (b) The growth rate of structure $f \sigma_{8}$ versus redshfit $z$ relation predicted from the Planck $\Lambda$ CDM model (shown as the solid black curve, with $1 \sigma$ and $2 \sigma$ uncertainties in gray); the existing measurements from previous redshift-space distortions from redshift surveys (6dFGS, 2dFGRS, SDSS, WiggleZ, VIPERS and BOSS); and the expected measurements from eBOSS. Also shown are curves corresponding to theories of gravity predicting growth going as $f=\Omega^{\gamma}$, with $\gamma$ differing slightly from the General Relativity value of 0.55 .

redshift, which constrains the equation of state of the universe. The prediction comes from the Planck ${ }^{87} \Lambda \mathrm{CDM}$ model, while the observed points are from previous BAO survey measurements by $6 \mathrm{dFGS},{ }^{52}$ WiggleZ $^{69}$ and SDSS/BOSS,${ }^{88,89}$ and a compilation of current SNe Ia measurements. ${ }^{90}$ The righthand panel of Figure 7 shows the predicted growth rate of structure $\left(f \sigma_{8}\right)$ as a function of redshift, and compares the Planck ${ }^{87} \Lambda$ CDM model to the measurements based on redshift-space distortions (RSD) from 6dFGS, ${ }^{53}$ 2dFGRS, ${ }^{30,91}$ SDSS LRGs, ${ }^{92}$ WiggleZ, ${ }^{78}$ VIPERS $^{93}$ and BOSS. ${ }^{84,94-97}$ Because the growth rate simultaneously tests both the cosmological model and the theory of gravity, the panel alsos shows the predictions for theories of gravity predicting growth going as $f=\Omega^{\gamma}$, with $\gamma$ differing slightly from the General Relativity value of 0.55 , demonstrating the sensitivity of these measurements to such alternatives.

All existing measurements are consistent with the standard $\Lambda \mathrm{CDM}$ model at the $5-10 \%$ level; the next generation of MOS surveys aim to tighten these constraints to a level approaching $1 \%$. The first of the new surveys is eBOSS, ${ }^{85,98}$ a 6-year survey (part of the SDSS-IV program) that started in 2014 and plans to complement and extend BOSS. It has the goal of measuring the distance-redshift relation from the BAO ruler with a precision of a few percent in each of four redshift bins over the range $0.6<z<2.2$. It will use four different tracers to cover this range: 250,000 luminous red galaxies with median redshift $z \approx 0.7 ; 195,000$ emission line galaxies with median redshift $z \approx 0.9 ; 500,000$ QSOs over $0.9<z<2.2$; and Lyman- $\alpha$ forest measurements using 120,000 QSOs at $z>2.1$. As well as determining the evolution of the geometry of the universe in order to determine the equation of state of dark energy, eBOSS aims to make stronger tests of General Relativity on cosmological scales through redshift-space distortion measurements, look for evidence of non-Gaussianity in the primordial density field, and tighten the constraints on the sum of the masses of all neutrino species. The constraints that eBOSS is predicted to yield ${ }^{85}$ on the comoving distance and the growth rate of structure as functions of redshift (assuming the Planck ${ }^{87} \Lambda \mathrm{CDM}$ model) are shown in Figure 7. 

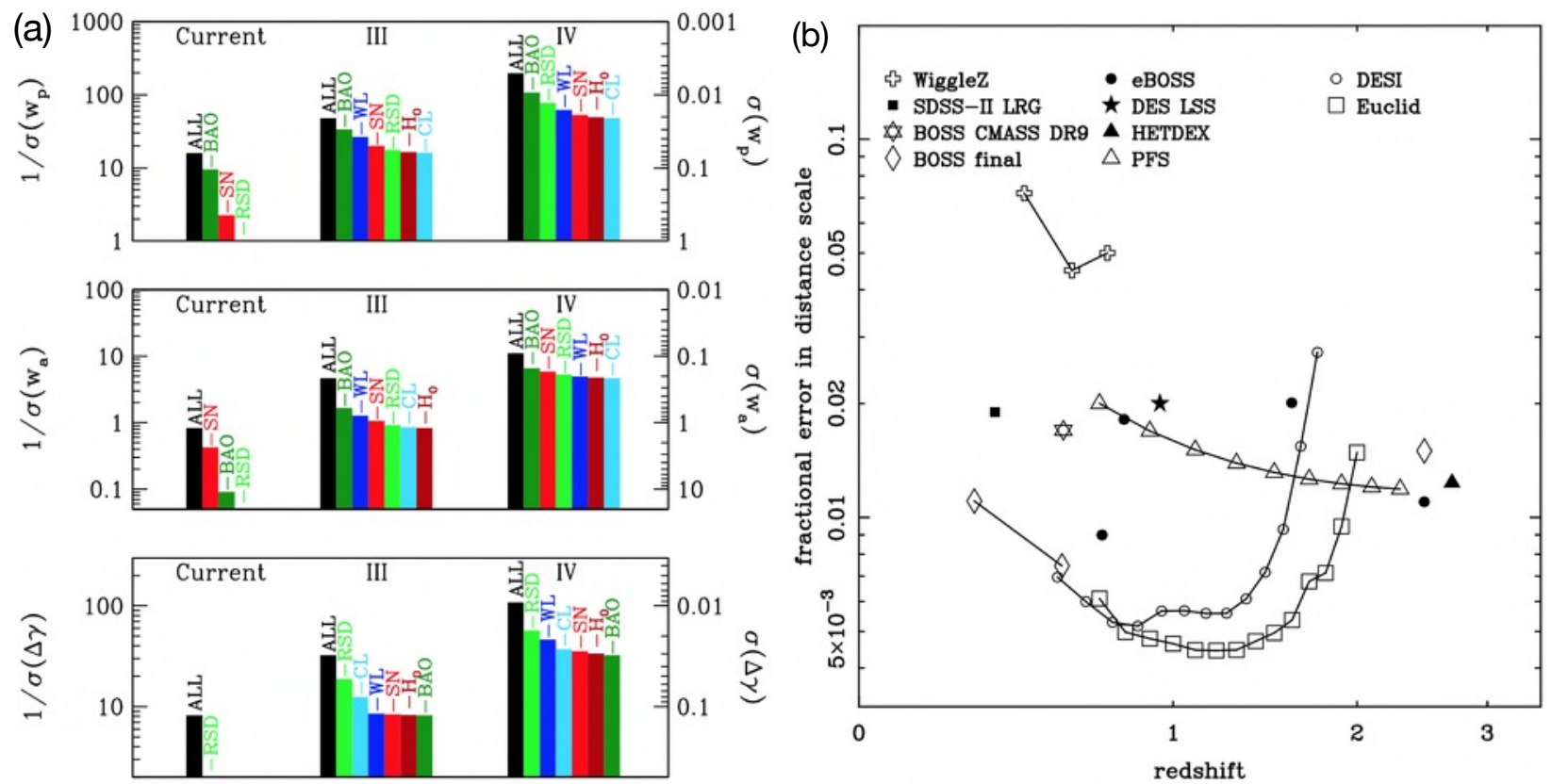

Figure 8. Current and predicted constraints from cosmological MOS surveys. (a) Potential improvements in figures of merit for constraints on the dark energy equation of state and the theory of gravity for existing and potential future BAO and RSD redshift surveys, and other methods. ${ }^{99}$ (b) The fractional error in constraints on the co-moving distance versus redshift relation, at various redshifts, from current and future MOS surveys. ${ }^{100}$

eBOSS is currently the state of the art as far as MOS cosmological surveys are concerned. However there are, of course, plans afoot for even more potent MOS surveys providing still greater discrimination between cosmological models and yet higher precision in determining cosmological parameters.

\section{THE FUTURE}

There are a number of proposed new cosmological MOS surveys planned for the near future, plus a wider variety of surveys aiming to explore the high-redshift universe using the next-generation Extremely Large Telescopes. The general program for these surveys is to continue the push for ever-larger samples over ever-larger volumes covering a wider range of redshfits. The focus of such surveys is ever-more-precise constraints on cosmological parameters, with a particular emphases on testing the nature of dark energy and the theory of gravity. Although all observations to date of the cosmic expansion history and the growth of structure are consistent with a flat $\Lambda$ CDM+GR model with $\Omega_{M} \approx 0.3$ and $\Omega_{\Lambda} \approx 0.7$, a number of plausible alternative models are also consistent with existing data. However BAO and RSD have to the potential, for sufficiently large surveys, to provide $\sim 10 \times$ stronger constraints on the equation of state of dark energy and the nature of the gravitational force. This opportunity is quantified by improvement in the relevant figures of merit, expressed as the inverse of the uncertainties on the $w_{a}$ and $w_{p}$ parameterisations of the DE equation of state and deviations $\Delta \gamma$ from the GR value of $\gamma=0.55$. These improvements for BAO and RSD measurements are shown for possible future MOS surveys $^{99}$ in Figure 8a; another view ${ }^{100}$ of the constraints, based on the actual and predicted fractional errors in the distance-redshift relation for existing and future MOS surveys, is provided in Figure 8b.

\subsection{Low Redshift Surveys}

At low redshifts, the Taipan survey ${ }^{101}$ on the newly-refurbished 1.2-metre UK Schmidt Telescope (UKST) aims to obtain high-precision constraints on the $z \approx 0$ values of key parameters such as the Hubble constant $\left(H_{0}\right)$ and the growth rate of structure. The survey will use the new TAIPAN ${ }^{102}$ fibre positioner and spectrograph, which are currently under construction at the Australian Astronomical Observatory. The TAIPAN positioner is a prototype for the MANIFEST ${ }^{103-105}$ fibre positioning system planned for the Giant Magellan Telescope (GMT), 

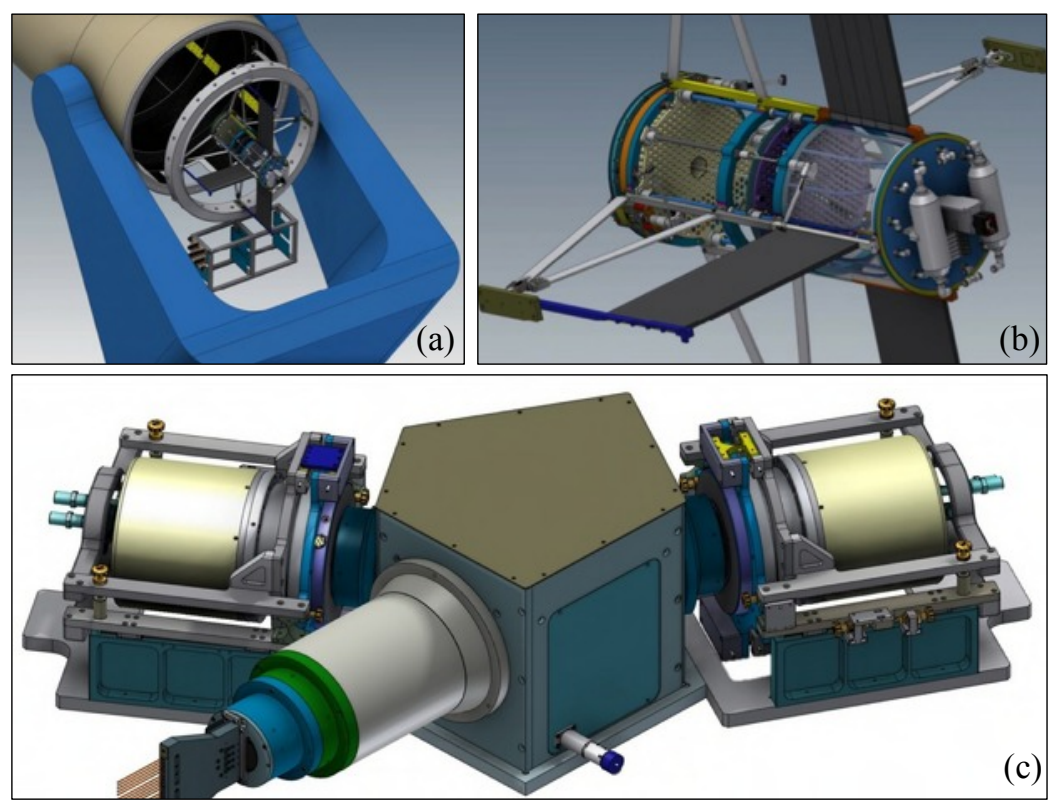

(c)

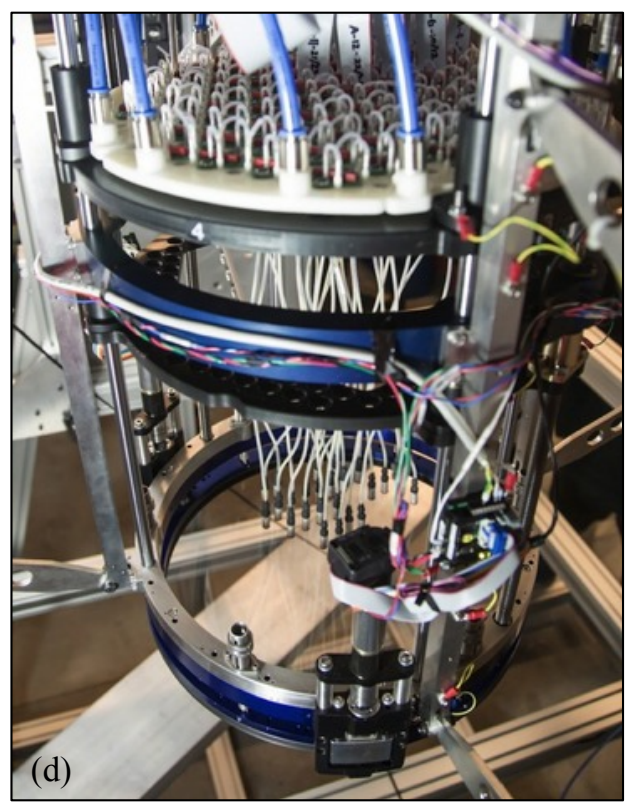

Figure 9. The components of the UKST+TAIPAN system: (a) the location of the TAIPAN fibre positioner within the UKST (in cutaway view); (b) a close-up rendering of the fibre positioner system containing the Starbugs; (c) the TAIPAN spectrograph layout, showing the blue and red channels covering the whole optical wavelength range; and (d) an image of some Starbugs being tested during the manufacturing process at AAO.

and uses autonomous piezo-electric micro-robots ('starbugs') to move the optical fibres about in the curved focal plane of the UKST. The TAIPAN spectrograph is a fixed-format, two-channel spectrograph covering the range $370-850 \mathrm{~nm}$ at $\mathrm{R}=2000-2400$ and delivering velocity resolution $\sigma_{v} \approx 60 \mathrm{~km} \mathrm{~s}^{-1}$ with a total efficiency of $\sim 30 \%$.

Covering essentially no redshift range, the Taipan survey cannot examine the evolution of such quantities, but it can measure them in the present-day universe without extrapolations from high redshift that require assumptions about the cosmological model. For example, the Planck ${ }^{87} \mathrm{CMB}$ observations yield a measurement of $H_{0}=67.3 \pm 0.7 \mathrm{~km} \mathrm{~s}^{-1} \mathrm{Mpc}^{-1}$, but this assumes a $\Lambda \mathrm{CDM}$ model with particular parameters in order to transform measurements made at $z \approx 1100$ to parameters at $z=0$. The Taipan survey, by contrast, will obtain redshifts for $\sim 1$ million galaxies at redshifts $z<0.2$ and directly measure $H_{0.1}$ with $1 \%$ precision from the BAO standard ruler that is imprinted in the large-scale structure. Taipan thus will provide a bookend measurement of the present-day expansion rate with a precision matching that of Planck's measurement of the expansion rate shortly after the Big Bang and that of ongoing and future cosmological surveys, such as the eBOSS and the Dark Energy Survey (DES), at high and intermediate redshifts. A recent review of the prospects in this field ${ }^{106}$ stated that: A measurement of the local value of $H_{0}$ to $1 \%$ precision and accuracy would provide key new insights into fundamental physics questions and lead to potentially revolutionary discoveries. While ongoing programs ${ }^{107}$ to measure $H_{0}$ at low redshift using supernovae as standard candles aim for a similar level of precision to Taipan, they have different and (arguably) greater systematic errors with which they must contend.

Similarly, Taipan is expected to obtain substantially better measurements at $z \approx 0$ of the growth rate of structure $\left(f \sigma_{8}\right)$ and the velocity field scaling parameter $(\beta)$, both of which should be determined to better than $5 \%$ precision. These low-redshift measurements are important because, again, they provide bookends to CMB measurements, but also because they depend on both the cosmological model and the theory of gravity, allowing tests of general relativity against alternative models. Combining Taipan's low-redshift constraints with higher-redshift MOS surveys and the CMB observations will significantly tighten the constraints on all important extensions to the standard cosmological model-in particular: the nature of dark energy and its evolution with time, the curvature of the universe as a test of inflationary models, the mass of neutrinos, and the total number of families of relativistic particles. ${ }^{99,108}$ 

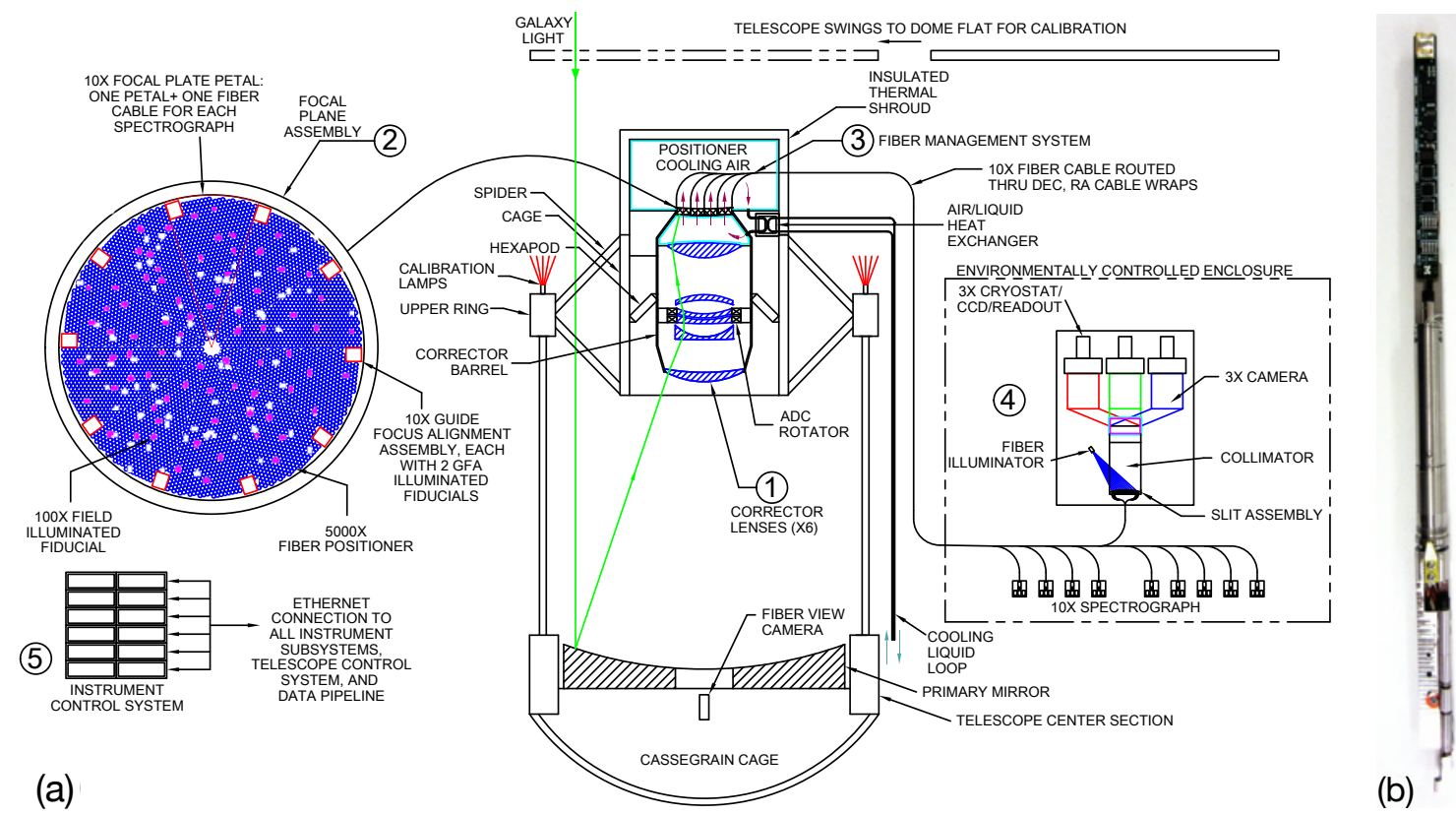

Figure 10. The DESI instrument. ${ }^{111}$ (a) Block diagram showing the main components of the instrument on the KPNO Mayall 4-metre telescope: 1. wide-field corrector and atmospheric dispersion compensator producing a 3.2-degree field of view at the telescope prime focus; 2. focal plane assembly, with 5000 fibers in 10 'petal' segments covering the field of view; 3. fiber management system and fiber cable run; 4. ten spectrographs, each taking 500 fibers from one of the 'petals' and each with three (blue/green/red) arms. (b) one of the robotic actuators for positioning individual fibres.

\subsection{Higher Redshift Surveys}

The primary focus of cosmological MOS surveys at higher redshifts is tracing the evolution of the geometry of the universe and the growth of large-scale structure over a period encompassing the epoch during which the universe changed from being matter-dominated to dark-energy-dominated (this occurs at $z \approx 0.8$ ). For direct redshift measurements, the upper limit on this range is set by the depth achievable with 4-metre and 8-metre telescopes under the constraint that for each redshift bin the survey must cover at least $\sim 1 \mathrm{Gpc}^{3}$ in order to determine the BAO signal, and hence the co-moving distance at that redshift, with sufficient precision to usefully constrain the dark energy equation of state. The most ambitious such survey currently planned is DESI, ${ }^{109,110}$ which aims to provide at least an order of magnitude improvement over BOSS/eBOSS, both in the comoving volume of the universe probed and in the total number of galaxies mapped. DESI is planned to start in 2018, with observations running for 5 years. It will be complementary to other planned new cosmological surveys such as those using the Large Synoptic Survey Telescope (LSST) and the EUCLID and WFIRST satellite missions.

The DESI instrument ${ }^{111}$ will be a fiber-fed spectrograph using a robotic positioner system and will be capable of taking up to 5000 simultaneous spectra over the range $360-980 \mathrm{~nm}$. The main components of the system, and one of the fiber positioners, are shown in Figure 10. The instrument will be installed at the prime focus of the 4-metre Mayall telescope at Kitt Peak and requires a new wide-field corrector and an atmospheric disperion compensator, which produce a 3.2-degree diameter field of view with an average scale of $14.1 \mathrm{arcsec} / \mathrm{mm}$. The 5000 fibers can be positioned over $7.5 \mathrm{deg}^{2}$ of the available $8.0 \mathrm{deg}^{2}$ in the field of view. The fiber density in the focal plane is $667 \mathrm{deg}^{-2}$ and the individual fibers have $107 \mu \mathrm{m}(1.5 \mathrm{arcsec})$ cores. The positioners are arrayed hexagonally, with a $10.4 \mathrm{~mm}(147 \mathrm{arcsec})$ pitch between fibers. Each positioner has two rotational degrees of freedom allowing it to reach any point within a $6 \mathrm{~mm}(85 \mathrm{arcsec})$ radius. The focal plane is divided into ten pie-slice-shaped petals containing 500 fibres, each feeding one of the ten spectrographs. Volume phase holographic gratings provide spectral dispersion in the three spectrograph channels $(360-593 \mathrm{~nm}, 566-772 \mathrm{~nm}$ and $747-980 \mathrm{~nm}$ ) with resolutions greater than 2000, 3200, and 4100 respectively. The blue arm of the spectrographs use CCDs from Imaging Technologies Lab, while the red and near-infrared channels use LBNL CCDs; all will 

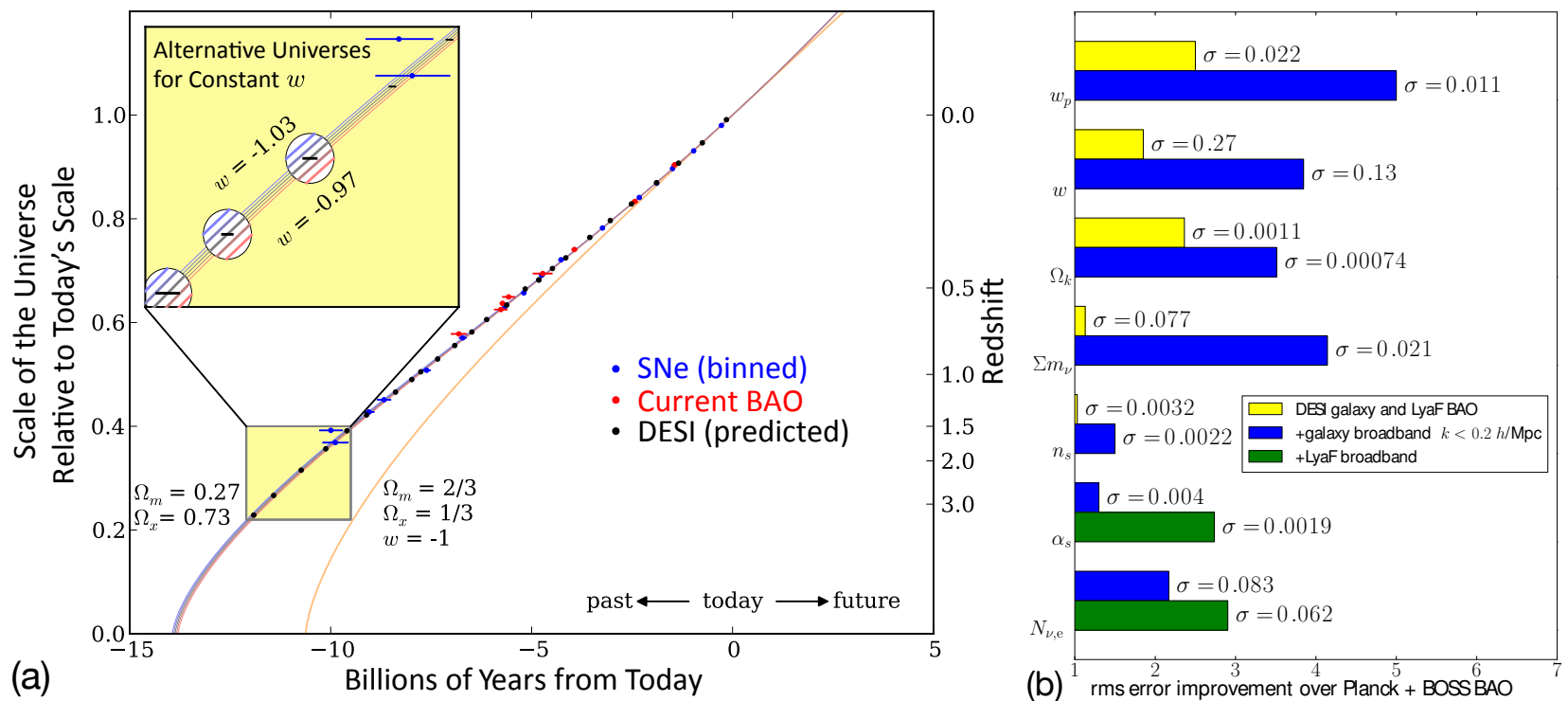

Figure 11. Predicted cosmological constraints and figures of merit for the DESI survey. ${ }^{110}$ (a) Measurements of the expansion rate, comparing DESI to the best current BAO and supernovae measurements. The inset illustrates the very high precision needed to distinguish models with constant $w$ ranging from 0.97 to 1.03 . (b) The improvements predicted for DESI in determining $w_{p}, w^{\prime}=w_{a}, \Omega_{k}, \sum m_{\nu}, n_{s}, \alpha_{s}$, and $N_{\nu, e}$, the number of neutrino-like (relativistic) species..

be $4 \mathrm{k} \times 4 \mathrm{k}$ with $15 \mu \mathrm{m}$ pixels. The system will have overall throughput around $35-40 \%$ over most of the spectral range, except at the bluest wavelengths.

The DESI instrument will be used to conduct a 5-year survey covering 14,000 deg $^{2}$ that will observe four classes of objects in order to map the matter distribution over the widest possible range of redshifts. ${ }^{110}$ At moderate redshifts (up to $z \approx 1$ ), DESI will observe luminous red galaxies (LRGs) in dark time and a magnitude-limited sample of up to 10 million galaxies with median redshift $z \approx 0.2$ in bright time. To map the matter distribution at higher redshifts, DESI will use emission line galaxies (ELGs, specifically those with strong [O II] lines) to reach $z \approx 1.7$. At the highest redshifts, DESI will use QSOs both as direct tracers of the matter distribution and, at $2.1<z<3.5$, as probes of the Ly- forest, tracing the distribution of neutral hydrogen. In total, DESI aims to obtain more than 30 million galaxy and QSO redshifts in order to measure the BAO feature and the matter power spectrum, including redshift space distortions, over most of cosmic history.

If DES achieves these observational goals, it will produce more than 30 separate measurements of the expansion rate of the universe, each with precision better than $1 \%$, over the redshift range $z=0.2$ to $z=3.5$. In terms of the Dark Energy Task Force figure of merit (FoM), which measures the combined precision on the dark energy equation of state today, $w_{0}$, and its evolution with redshift, $w_{a}$, DESI's galaxy BAO measurements are predicted to achieve a FoM of 133, which is more than $3 \times$ better than the FoM for all previous galaxy BAO measurements combined. If the Lyman- $\alpha$ forest measurements are also included, then DESI's predicted FoM increases to 169. Finally, including galaxy broadband power spectrum measurements for wavenumbers $k<0.1 \mathrm{~h} \mathrm{Mpc}{ }^{1}$, DESI's FoM rises still further to 332 , and to 704 if it is possible to obtain reliable measurements for $k<0.2 \mathrm{~h} \mathrm{Mpc}^{1}$.

In addition to providing vastly improved constraints on dark energy, DESI will also measure the sum of neutrino masses with an uncertainty of just $0.02 \mathrm{eV}$ (again, if the power spectrum is measured reliably for $k<0.2 \mathrm{~h} \mathrm{Mpc}^{1}$ ). This should be sufficient to make the first direct detection of the sum of the neutrino masses at $3 \sigma$ significance and to rule out the the inverted mass hierarchy with $99 \%$ confidence (if the hierarchy is normal and the masses are minimal). The survey will also place tighter constraints on alternative (non-GR) theories of gravity and on models for inflation by measuring the spectral index $n_{s}$ and its running with wavenumber, $\alpha_{s}$, and also the velocity fields of in the infall regions around clusters of galaxies. The cosmological constraints that DESI is predicted to achieve, and the gains relative to existing results, are summarized in Figure 11. 
Other facilities on 4-metre and 8-metre telescopes that have the capability to carry out similar surveys include: WEAVE $^{112}$ on the William Herschel 4.2-metre telescope at La Palma Observatory (1000 fibers over a 3 deg $^{2}$ field from 2017); 4MOST ${ }^{113}$ on ESO's 4.1-metre VISTA facility at Paranal (2400 fibers over a $4 \mathrm{deg}^{2}$ field from 2021); and the Prime Focus Spectrograph ${ }^{114}$ (PFS) on the Subaru 8.2-metre telescope on Mauna Kea (2400 fibers over a $1.3 \mathrm{deg}^{2}$ field from 2019). However these facilities generally have much broader scientific goals than just cosmology surveys, and often are general-use facilities rather than dedicated survey facilities.

\subsection{ELT Surveys}

At higher redshifts, cosmological surveys such as BOSS and DESI use the Lyman- $\alpha$ forest in quasar spectra to map large-scale structure and place constraints on the geometry of the universe and the growth of large-scale structure just as galaxy redshift surveys do at lower redshifts. This approach will be even more powerful for the new generation of Extremely Large Telescopes (ELTs). With collecting areas 6-15 times greater than the largest existing telescopes, ELTs can use substantially fainter (and therefore much more common) background sources such as Lyman-break galaxies to illuminate the Lyman- $\alpha$ forest. This means ELTs will be able to probe the matter distribution on much shorter length scales than surveys using quasars and build much larger samples. The disadvantage of ELTs is that they have significantly smaller fields of view than the wide-field 4-metre telescopes (2-3 deg diameter) and 8-metre telescopes (up to 1.5 deg diameter).

The largest ELT field of view is the 20 arcmin diameter provided by the 25-metre Giant Magellan Telescope (GMT). This field will be fully exploited by the MANIFEST fiber system ${ }^{103-105}$ (see also the paper by Lawrence et al. in these proceedings), which will use starbugs (as on the prototype TAIPAN system on the UKST) to position hundreds of small integral field units (effectively, image-slicers). MANIFEST will feed both the GMACS medium-resolution optical spectrograph and the G-CLEF high-resolution optical spectrograph (see paper by Jacoby et al. in these proceedings). The image-slicing capability means that MANIFEST more than doubles the normal slit-limited spectral resolution of GMACS, providing a much better match to the resolved velocity structure in the Lyman- $\alpha$ forest.

GMT+MANIFEST could be used to perform a large survey to construct a 3D map of the IGM, using Lyman break galaxies (LBGs) as background sources with which to probe the Lyman- $\alpha$ forest. The crucial wavelength range for such a survey is $0.36-0.56 \mu \mathrm{m}$, corresponding to a redshift range $2.0<z<3.5$. The intrinsic size of the LBGs is approximately $2 \mathrm{kpc}$ or $0.3 \mathrm{arcsec}$, so they are well matched to the fiber sampling of MANIFEST, with each starbug having 17 fibers each sampling 0.2 arcsec. With 4-hour exposures, GMT+MANIFEST can achieve sufficient $\mathrm{S} / \mathrm{N}$ for targets at $r \approx 25$, enabling a $\sim 1 \mathrm{deg}^{2}$ survey of LBG targets (with a surface density of $\sim 4000$ $\operatorname{deg}^{-2}$ to $r \approx 24$ ) to be achieved in about 20 nights. This would be complemented by a sparsely-sampled galaxy redshift survey of the same area, to a depth of $r \approx 26$ (corresponding to a surface density of $\sim 30,000 \mathrm{deg}^{-2}$ ), taking about 40 nights. Such a combined survey would provide constraints on the growth of small-scale structure at high redshifts that are not achievable with 8-metre class telescopes that have to use brighter (and sparser) QSOs as the background sources for the Lyman- $\alpha$ forest.

In the ELT era, the greatest advantage of 8-metre-class telescopes will be their relatively large fields of view. Thus, like 4-metre telescopes in the 8-metre era, 8-metre telescopes in the ELT era will have strong reasons to develop wide-field capabilities. Since LSST will dominate the 8-metre imaging niche, other 8-metre telescopes will likely seek to develop some variety of wide-field MOS capability as their cutting-edge instrumentation, as Subaru is already doing with PFS.

\section{CONCLUSIONS}

The contributions of multi-object spectroscopy to cosmology over the past two decades have been profound. Redshift surveys of large-scale structure are one of the pillars supporting modern precision cosmology, and have considerable potential to increase their precision and range of applicability. MOS instruments on 4-metre, 8metre, and the next generation of extremely large telescopes will continue to be powerful tools for determining the evolution of the universe and attacking the fundamental question of the nature of dark energy. 


\section{ACKNOWLEDGMENTS}

This work supported in part by Australian Research Council grants LE140100052 \& DP160102075. The author thanks his collaborators on the 2dFGRS, 6dFGS, WiggleZ, and Taipan science teams and on the 2dF, 6dF, OzPoz, TAIPAN and MANIFEST instrument teams for making the last 20 years both productive and pleasurable.

\section{REFERENCES}

[1] Colless, M. and Hewett, P., "The dynamics of rich clusters. I - Velocity data," Monthly Notices of the Royal Astronomical Society 224, 453-472 (Jan. 1987).

[2] Broadhurst, T. J., Ellis, R. S., and Shanks, T., "The Durham/Anglo-Australian Telescope faint galaxy redshift survey," Monthly Notices of the Royal Astronomical Society 235, 827-856 (Dec. 1988).

[3] Colless, M., Ellis, R. S., Taylor, K., and Hook, R. N., "The LDSS deep redshift survey," Monthly Notices of the Royal Astronomical Society 244, 408-423 (June 1990).

[4] Ellis, R. S., Colless, M., Broadhurst, T., Heyl, J., and Glazebrook, K., "Autofib Redshift Survey - I. Evolution of the galaxy luminosity function," Monthly Notices of the Royal Astronomical Society 280, 235-251 (May 1996).

[5] de Lapparent, V., Geller, M. J., and Huchra, J. P., "A slice of the universe," Astrophysical Journal Letters 302, L1-L5 (Mar. 1986).

[6] Geller, M. J. and Huchra, J. P., "Mapping the universe," Science 246, 897-903 (Nov. 1989).

[7] de Lapparent, V., Geller, M. J., and Huchra, J. P., "Measures of large-scale structure in the CfA redshift survey slices," Astrophysical Journal 369, 273-286 (Mar. 1991).

[8] Kodama, H. and Sasaki, M., "Cosmological Perturbation Theory," Progress of Theoretical Physics Supplement 78, 1 (1984).

[9] Mukhanov, V. F., Feldman, H. A., and Brandenberger, R. H., "Theory of cosmological perturbations," Physics Reports 215, 203-333 (June 1992).

[10] Shectman, S. A., Landy, S. D., Oemler, A., et al., "The Las Campanas Redshift Survey," Astrophysical Journal 470, 172 (Oct. 1996).

[11] Landy, S. D., Shectman, S. A., Lin, H., et al., "The Two-dimensional Power Spectrum of the Las Campanas Redshift Survey: Detection of Excess Power on 100 H -1 MPC Scales," Astrophysical Journal Letters 456, L1 (Jan. 1996).

[12] Lin, H., Kirshner, R. P., Shectman, S. A., et al., "The Power Spectrum of Galaxy Clustering in the Las Campanas Redshift Survey," Astrophysical Journal 471, 617 (Nov. 1996).

[13] Tucker, D. L., Oemler, Jr., A., Kirshner, R. P., et al., "The Las Campanas Redshift Survey galaxy-galaxy autocorrelation function," Monthly Notices of the Royal Astronomical Society 285, L5-L9 (Feb. 1997).

[14] Landy, S. D., Szalay, A. S., and Broadhurst, T. J., "The Pairwise Velocity Distribution of Galaxies in the Las Campanas Redshift Survey," Astrophysical Journal Letters 494, L133-L136 (Feb. 1998).

[15] Canavezes, A., Springel, V., Oliver, S. J., et al., "The topology of the IRAS Point Source Catalogue Redshift Survey," Monthly Notices of the Royal Astronomical Society 297, 777-793 (July 1998).

[16] Tadros, H., Ballinger, W. E., Taylor, A. N., et al., "Spherical harmonic analysis of the PSCz galaxy catalogue: redshift distortions and the real-space power spectrum," Monthly Notices of the Royal Astronomical Society 305, 527-546 (May 1999).

[17] Schmoldt, I. M., Saar, V., Saha, P., et al., "On Density and Velocity Fields and beta from the IRAS PSCZ Survey," Astronomical Journal 118, 1146-1160 (Sept. 1999).

[18] Rowan-Robinson, M., Sharpe, J., Oliver, S. J., et al., "The IRAS PSCz dipole," Monthly Notices of the Royal Astronomical Society 314, 375-397 (May 2000).

[19] Saunders, W., Sutherland, W. J., Maddox, S. J., et al., "The PSCz catalogue," Monthly Notices of the Royal Astronomical Society 317, 55-63 (Sept. 2000).

[20] Cannon, R., "Some comments on large telescope astronomy in Britain and Australia," Astrophysics 8 Space Science 160, 275-280 (Oct. 1989).

[21] Taylor, K. and Gray, P., "Design study for a new wide-field AAT Prime-focus - The 2dF," in Advanced Technology Optical Telescopes IV, Barr, L. D., ed., Proceedings of the SPIE 1236, 290-299 (July 1990). 
[22] Gray, P. and Taylor, K., "Fibre optic instrumentation for the wide-field AAT prime-focus - The 2dF," in Instrumentation in Astronomy VII, Crawford, D. L., ed., Proceedings of the SPIE 1235, 709-720 (July 1990).

[23] Lewis, I. J., Cannon, R. D., Taylor, K., et al., "The Anglo-Australian Observatory 2dF facility," Monthly Notices of the Royal Astronomical Society 333, 279-299 (June 2002).

[24] Colless, M., Dalton, G., Maddox, S., et al., "The 2dF Galaxy Redshift Survey: spectra and redshifts," Monthly Notices of the Royal Astronomical Society 328, 1039-1063 (Dec. 2001).

[25] Colless, M., Peterson, B. A., Jackson, C., et al., "The 2dF Galaxy Redshift Survey: Final Data Release," ArXiv Astrophysics e-prints (June 2003).

[26] Peacock, J. A., Cole, S., Norberg, P., et al., "A measurement of the cosmological mass density from clustering in the 2dF Galaxy Redshift Survey," Nature 410, 169-173 (Mar. 2001).

[27] Percival, W. J., Baugh, C. M., Bland-Hawthorn, J., et al., "The 2dF Galaxy Redshift Survey: the power spectrum and the matter content of the Universe," Monthly Notices of the Royal Astronomical Society 327, 1297-1306 (Nov. 2001).

[28] Efstathiou, G., Moody, S., Peacock, J. A., et al., "Evidence for a non-zero $\Lambda$ and a low matter density from a combined analysis of the 2dF Galaxy Redshift Survey and cosmic microwave background anisotropies," Monthly Notices of the Royal Astronomical Society 330, L29-L35 (Feb. 2002).

[29] Elgarøy, Ø., Lahav, O., Percival, W. J., et al., "New Upper Limit on the Total Neutrino Mass from the 2 Degree Field Galaxy Redshift Survey," Physical Review Letters 89, 061301 (July 2002).

[30] Cole, S., Percival, W. J., Peacock, J. A., et al., "The 2dF Galaxy Redshift Survey: power-spectrum analysis of the final data set and cosmological implications," Monthly Notices of the Royal Astronomical Society 362, 505-534 (Sept. 2005).

[31] Colless, M., "The 2dF Galaxy Redshift Survey," in Celebrating the AAO: Past, Present, Future, Cannon, R. and Malin, D., eds., 239-247, Commonwealth of Australia (2011).

[32] York, D. G., Adelman, J., Anderson, Jr., J. E., et al., "The Sloan Digital Sky Survey: Technical Summary," Astronomical Journal 120, 1579-1587 (Sept. 2000).

[33] Stoughton, C., Lupton, R. H., Bernardi, M., et al., "Sloan Digital Sky Survey: Early Data Release," Astronomical Journal 123, 485-548 (Jan. 2002).

[34] Smee, S. A., Gunn, J. E., Uomoto, A., et al., "The Multi-object, Fiber-fed Spectrographs for the Sloan Digital Sky Survey and the Baryon Oscillation Spectroscopic Survey," Astronomical Journal 146, 32 (Aug. 2013).

[35] Abazajian, K. N., Adelman-McCarthy, J. K., Agüeros, M. A., et al., "The Seventh Data Release of the Sloan Digital Sky Survey," Astrophysical Journal Supplement 182, 543-558 (June 2009).

[36] Zehavi, I., Blanton, M. R., Frieman, J. A., et al., "Galaxy Clustering in Early Sloan Digital Sky Survey Redshift Data," Astrophysical Journal 571, 172-190 (May 2002).

[37] Tegmark, M., Blanton, M. R., Strauss, M. A., et al., "The Three-Dimensional Power Spectrum of Galaxies from the Sloan Digital Sky Survey," Astrophysical Journal 606, 702-740 (May 2004).

[38] Eisenstein, D. J., Zehavi, I., Hogg, D. W., et al., "Detection of the Baryon Acoustic Peak in the Large-Scale Correlation Function of SDSS Luminous Red Galaxies," Astrophysical Journal 633, 560-574 (Nov. 2005).

[39] Percival, W. J., Nichol, R. C., Eisenstein, D. J., et al., "The Shape of the Sloan Digital Sky Survey Data Release 5 Galaxy Power Spectrum," Astrophysical Journal 657, 645-663 (Mar. 2007).

[40] Wakamatsu, K., Colless, M., Jarrett, T., et al., "The 6dF Galaxy Survey," in The Proceedings of the IAU 8th Asian-Pacific Regional Meeting, Volume 1, Ikeuchi, S., Hearnshaw, J., and Hanawa, T., eds., Astronomical Society of the Pacific Conference Series 289, 97-104 (May 2003).

[41] Jones, D. H., Saunders, W., Colless, M., et al., "The 6dF Galaxy Survey: samples, observational techniques and the first data release," Monthly Notices of the Royal Astronomical Society 355, 747-763 (Dec. 2004).

[42] Colless, M., Jones, H., Campbell, L., et al., "The 6dF Galaxy Survey: Mass and Motions in the Local Universe," in Maps of the Cosmos, Colless, M., Staveley-Smith, L., and Stathakis, R. A., eds., IAU Symposium 216, 180 (Jan. 2005). 
[43] Colless, M. and Jones, D. H., "The 6dF Galaxy Survey of the Local Universe," in The 9th Asian-Pacific Regional IAU Meeting, Sutantyo, W., Premadi, P. W., Mahasena, P., Hidayat, T., and Mineshige, S., eds., 200 (Jan. 2006).

[44] Jones, D. H., Read, M. A., Saunders, W., et al., "The 6dF Galaxy Survey: final redshift release (DR3) and southern large-scale structures," Monthly Notices of the Royal Astronomical Society 399, 683-698 (Oct. 2009).

[45] Magoulas, C., Springob, C. M., Colless, M., et al., "The 6dF Galaxy Survey: the near-infrared Fundamental Plane of early-type galaxies," Monthly Notices of the Royal Astronomical Society 427, 245-273 (Nov. 2012).

[46] Campbell, L. A., Lucey, J. R., Colless, M., et al., "The 6dF Galaxy Survey: Fundamental Plane data," Monthly Notices of the Royal Astronomical Society 443, 1231-1251 (Sept. 2014).

[47] Springob, C. M., Magoulas, C., Colless, M., et al., "The 6dF Galaxy Survey: peculiar velocity field and cosmography," Monthly Notices of the Royal Astronomical Society 445, 2677-2697 (Dec. 2014).

[48] Parker, Q. A., Watson, F. G., and Miziarski, S., "6dF: an Automated Multi-Object Fiber Spectroscopy System for the UKST," in Fiber Optics in Astronomy III, Arribas, S., Mediavilla, E., and Watson, F., eds., Astronomical Society of the Pacific Conference Series 152, 80 (1998).

[49] Watson, F. G., Parker, Q. A., and Miziarski, S., "6dF: a very efficient multiobject spectroscopy system for the UK Schmidt Telescope," in Optical Astronomical Instrumentation, D'Odorico, S., ed., Proceedings of the SPIE 3355, 834-843 (July 1998).

[50] Parker, Q. A. and Watson, F., "6dF: A new spectroscopic survey facility for the U.K. Schmidt Telescope," IAU Commission on Instruments 12, 5 (2000).

[51] Watson, F. G., Parker, Q. A., Bogatu, G., et al., "Progress with 6dF: a multi-object spectroscopy system for all-sky surveys," in Optical and IR Telescope Instrumentation and Detectors, Iye, M. and Moorwood, A. F., eds., Proceedings of the SPIE 4008, 123-128 (Aug. 2000).

[52] Beutler, F., Blake, C., Colless, M., et al., "The 6dF Galaxy Survey: baryon acoustic oscillations and the local Hubble constant," Monthly Notices of the Royal Astronomical Society 416, 3017-3032 (Oct. 2011).

[53] Beutler, F., Blake, C., Colless, M., et al., "The 6dF Galaxy Survey: $\mathrm{z} \approx 0$ measurements of the growth rate and $\sigma_{8}, "$ Monthly Notices of the Royal Astronomical Society 423, 3430-3444 (July 2012).

[54] Scrimgeour, M. I., Davis, T. M., Blake, C., et al., "The 6dF Galaxy Survey: bulk flows on 50-70 h ${ }^{-1} \mathrm{Mpc}$ scales," Monthly Notices of the Royal Astronomical Society 455, 386-401 (Jan. 2016).

[55] Magoulas, C., Colless, M., Springob, C. M., et al., "The 6dF Galaxy Survey: bulk flows and $\beta$ from fitting the peculiar velocity field," in prep. (2016).

[56] Johnson, A., Blake, C., Koda, J., et al., "The 6dF Galaxy Survey: cosmological constraints from the velocity power spectrum," Monthly Notices of the Royal Astronomical Society 444, 3926-3947 (Nov. 2014).

[57] Saunders, W., Bridges, T., Gillingham, P., et al., "AAOmega: a scientific and optical overview," in Groundbased Instrumentation for Astronomy, Moorwood, A. F. M. and Iye, M., eds., Proceedings of the SPIE 5492, 389-400 (Sept. 2004).

[58] Smith, G. A., Saunders, W., Bridges, T., et al., "AAOmega: a multipurpose fiber-fed spectrograph for the AAT," in Ground-based Instrumentation for Astronomy, Moorwood, A. F. M. and Iye, M., eds., Proceedings of the SPIE 5492, 410-420 (Sept. 2004).

[59] Sharp, R., Saunders, W., Smith, G., et al., "Performance of AAOmega: the AAT multi-purpose fiber-fed spectrograph," in Society of Photo-Optical Instrumentation Engineers (SPIE) Conference Series, Proceedings of the SPIE 6269, 62690G (June 2006).

[60] Ellis, S. C., Ireland, M., Lawrence, J. S., et al., "KOALA: a wide-field 1000 element integral-field unit for the Anglo-Australian Telescope," in Ground-based and Airborne Instrumentation for Astronomy IV, Proceedings of the SPIE 8446, 84460V (Sept. 2012).

[61] Zhelem, R., Brzeski, J., Case, S., et al., "KOALA, a wide-field 1000 element integral-field unit for the AngloAustralian Telescope: assembly and commissioning," in Ground-based and Airborne Instrumentation for Astronomy V, Proceedings of the SPIE 9147, 91473K (July 2014).

[62] Croom, S. M., Lawrence, J. S., Bland-Hawthorn, J., et al., "The Sydney-AAO Multi-object Integral field spectrograph," Monthly Notices of the Royal Astronomical Society 421, 872-893 (Mar. 2012). 
[63] Bryant, J. J., Owers, M. S., Robotham, A. S. G., et al., "The SAMI Galaxy Survey: instrument specification and target selection," Monthly Notices of the Royal Astronomical Society 447, 2857-2879 (Mar. 2015).

[64] Driver, S. P., Hill, D. T., Kelvin, L. S., et al., "Galaxy and Mass Assembly (GAMA): survey diagnostics and core data release," Monthly Notices of the Royal Astronomical Society 413, 971-995 (May 2011).

[65] Allen, J. T., Croom, S. M., Konstantopoulos, I. S., et al., "The SAMI Galaxy Survey: Early Data Release," Monthly Notices of the Royal Astronomical Society 446, 1567-1583 (Jan. 2015).

[66] Cannon, R., Drinkwater, M., Edge, A., et al., "The 2dF-SDSS LRG and QSO (2SLAQ) Luminous Red Galaxy Survey," Monthly Notices of the Royal Astronomical Society 372, 425-442 (Oct. 2006).

[67] Drinkwater, M. J., Jurek, R. J., Blake, C., et al., "The WiggleZ Dark Energy Survey: survey design and first data release," Monthly Notices of the Royal Astronomical Society 401, 1429-1452 (Jan. 2010).

[68] Yuan, F., Lidman, C., Davis, T. M., et al., "OzDES multifibre spectroscopy for the Dark Energy Survey: first-year operation and results," Monthly Notices of the Royal Astronomical Society 452, 3047-3063 (Sept. 2015).

[69] Parkinson, D., Riemer-Sørensen, S., Blake, C., et al., "The WiggleZ Dark Energy Survey: Final data release and cosmological results," Physics Review D 86, 103518 (Nov. 2012).

[70] Blake, C., Davis, T., Poole, G. B., et al., "The WiggleZ Dark Energy Survey: testing the cosmological model with baryon acoustic oscillations at $\mathrm{z}=0.6$," Monthly Notices of the Royal Astronomical Society 415, 2892-2909 (Aug. 2011).

[71] Blake, C., Kazin, E. A., Beutler, F., et al., "The WiggleZ Dark Energy Survey: mapping the distanceredshift relation with baryon acoustic oscillations," Monthly Notices of the Royal Astronomical Society 418, 1707-1724 (Dec. 2011).

[72] Blake, C., Glazebrook, K., Davis, T. M., et al., "The WiggleZ Dark Energy Survey: measuring the cosmic expansion history using the Alcock-Paczynski test and distant supernovae," Monthly Notices of the Royal Astronomical Society 418, 1725-1735 (Dec. 2011).

[73] Kazin, E. A., Koda, J., Blake, C., et al., "The WiggleZ Dark Energy Survey: improved distance measurements to $\mathrm{z}=1$ with reconstruction of the baryonic acoustic feature," Monthly Notices of the Royal Astronomical Society 441, 3524-3542 (July 2014).

[74] Blake, C., Brough, S., Colless, M., et al., "The WiggleZ Dark Energy Survey: the selection function and $\mathrm{z}=0.6$ galaxy power spectrum," Monthly Notices of the Royal Astronomical Society 406, 803-821 (Aug. 2010).

[75] Blake, C., Brough, S., Colless, M., et al., "The WiggleZ Dark Energy Survey: the growth rate of cosmic structure since redshift z=0.9," Monthly Notices of the Royal Astronomical Society 415, 2876-2891 (Aug. 2011).

[76] Contreras, C., Blake, C., Poole, G. B., et al., "The WiggleZ Dark Energy Survey: measuring the cosmic growth rate with the two-point galaxy correlation function," Monthly Notices of the Royal Astronomical Society 430, 924-933 (Apr. 2013).

[77] Marín, F. A., Blake, C., Poole, G. B., et al., "The WiggleZ Dark Energy Survey: constraining galaxy bias and cosmic growth with three-point correlation functions," Monthly Notices of the Royal Astronomical Society 432, 2654-2668 (July 2013).

[78] Blake, C., Brough, S., Colless, M., et al., "The WiggleZ Dark Energy Survey: joint measurements of the expansion and growth history at $\mathrm{z}<1$, , Monthly Notices of the Royal Astronomical Society 425, 405-414 (Sept. 2012).

[79] Riemer-Sørensen, S., Blake, C., Parkinson, D., et al., "WiggleZ Dark Energy Survey: Cosmological neutrino mass constraint from blue high-redshift galaxies," Physics Review D 85, 081101 (Apr. 2012).

[80] Scrimgeour, M. I., Davis, T., Blake, C., et al., "The WiggleZ Dark Energy Survey: the transition to large-scale cosmic homogeneity," Monthly Notices of the Royal Astronomical Society 425, 116-134 (Sept. 2012).

[81] Eisenstein, D. J., Weinberg, D. H., Agol, E., et al., "SDSS-III: Massive Spectroscopic Surveys of the Distant Universe, the Milky Way, and Extra-Solar Planetary Systems," Astronomical Journal 142, 72 (Sept. 2011). 
[82] Ahn, C. P., Alexandroff, R., Allende Prieto, C., et al., "The Ninth Data Release of the Sloan Digital Sky Survey: First Spectroscopic Data from the SDSS-III Baryon Oscillation Spectroscopic Survey," Astrophysical Journal Supplement 203, 21 (Dec. 2012).

[83] Dawson, K. S., Schlegel, D. J., Ahn, C. P., et al., "The Baryon Oscillation Spectroscopic Survey of SDSSIII," Astronomical Journal 145, 10 (Jan. 2013).

[84] Alam, S., Albareti, F. D., Allende Prieto, C., et al., "The Eleventh and Twelfth Data Releases of the Sloan Digital Sky Survey: Final Data from SDSS-III," Astrophysical Journal Supplement 219, 12 (July 2015).

[85] Dawson, K. S., Kneib, J.-P., Percival, W. J., et al., "The SDSS-IV Extended Baryon Oscillation Spectroscopic Survey: Overview and Early Data," Astronomical Journal 151, 44 (Feb. 2016).

[86] Bundy, K., Bershady, M. A., Law, D. R., et al., "Overview of the SDSS-IV MaNGA Survey: Mapping nearby Galaxies at Apache Point Observatory," Astrophysical Journal 798, 7 (Jan. 2015).

[87] Planck Collaboration, Ade, P. A. R., Aghanim, N., et al., "Planck 2015 results. XIII. Cosmological parameters," ArXiv e-prints (Feb. 2015).

[88] Xu, X., Cuesta, A. J., Padmanabhan, N., Eisenstein, D. J., and McBride, C. K., "Measuring $\mathrm{D}_{A}$ and $\mathrm{H}$ at $\mathrm{z}=0.35$ from the SDSS DR7 LRGs using baryon acoustic oscillations," Monthly Notices of the Royal Astronomical Society 431, 2834-2860 (May 2013).

[89] Ross, A. J., Samushia, L., Howlett, C., et al., "The clustering of the SDSS DR7 main Galaxy sample - I. A 4 per cent distance measure at $\mathrm{z}=0.15, "$ Monthly Notices of the Royal Astronomical Society 449, 835-847 (May 2015).

[90] Betoule, M., Kessler, R., Guy, J., et al., "Improved cosmological constraints from a joint analysis of the SDSS-II and SNLS supernova samples," Astronomy 83 Astrophysics 568, A22 (Aug. 2014).

[91] Song, Y.-S. and Percival, W. J., "Reconstructing the history of structure formation using redshift distortions," Journal of Cosmology and Astroparticle Physics 10, 004 (Oct. 2009).

[92] Oka, A., Saito, S., Nishimichi, T., Taruya, A., and Yamamoto, K., "Simultaneous constraints on the growth of structure and cosmic expansion from the multipole power spectra of the SDSS DR7 LRG sample," Monthly Notices of the Royal Astronomical Society 439, 2515-2530 (Apr. 2014).

[93] de la Torre, S., Guzzo, L., Peacock, J. A., et al., "The VIMOS Public Extragalactic Redshift Survey (VIPERS) . Galaxy clustering and redshift-space distortions at $\mathrm{z} \approx 0.8$ in the first data release," Astronomy E Astrophysics 557, A54 (Sept. 2013).

[94] Chuang, C.-H., Prada, F., Cuesta, A. J., et al., "The clustering of galaxies in the SDSS-III Baryon Oscillation Spectroscopic Survey: single-probe measurements and the strong power of $\mathrm{f}(\mathrm{z}) \sigma_{8}(\mathrm{z})$ on constraining dark energy," Monthly Notices of the Royal Astronomical Society 433, 3559-3571 (Aug. 2013).

[95] Beutler, F., Saito, S., Seo, H.-J., et al., "The clustering of galaxies in the SDSS-III Baryon Oscillation Spectroscopic Survey: testing gravity with redshift space distortions using the power spectrum multipoles," Monthly Notices of the Royal Astronomical Society 443, 1065-1089 (Sept. 2014).

[96] Reid, B. A., Seo, H.-J., Leauthaud, A., Tinker, J. L., and White, M., "A 2.5 per cent measurement of the growth rate from small-scale redshift space clustering of SDSS-III CMASS galaxies," Monthly Notices of the Royal Astronomical Society 444, 476-502 (Oct. 2014).

[97] Samushia, L., Reid, B. A., White, M., et al., "The clustering of galaxies in the SDSS-III Baryon Oscillation Spectroscopic Survey: measuring growth rate and geometry with anisotropic clustering," Monthly Notices of the Royal Astronomical Society 439, 3504-3519 (Apr. 2014).

[98] Zhao, G.-B., Wang, Y., Ross, A. J., et al., "The extended Baryon Oscillation Spectroscopic Survey: a cosmological forecast," Monthly Notices of the Royal Astronomical Society 457, 2377-2390 (Apr. 2016).

[99] Weinberg, D. H., Mortonson, M. J., Eisenstein, D. J., et al., "Observational probes of cosmic acceleration," Physics Reports 530, 87-255 (Sept. 2013).

[100] Percival, W. J., "Large Scale Structure Observations," ArXiv e-prints (Dec. 2013).

[101] Colless, M., Beutler, F., and Blake, C., "Measuring $\mathrm{H}_{0}$ from the 6dF Galaxy Survey and future low-redshift surveys," in Advancing the Physics of Cosmic Distances, de Grijs, R., ed., IAU Symposium 289, 319-322 (Feb. 2013).

[102] Kuehn, K., Lawrence, J., Brown, D. M., et al., "TAIPAN: optical spectroscopy with StarBugs," in Groundbased and Airborne Instrumentation for Astronomy V, Proceedings of the SPIE 9147, 914710 (July 2014). 
[103] Saunders, W., Colless, M., Saunders, I., et al., "MANIFEST: a many-instrument fiber-positioning system for GMT," in Ground-based and Airborne Instrumentation for Astronomy III, Proceedings of the SPIE 7735, 773568 (July 2010).

[104] Goodwin, M., Brzeski, J., Case, S., et al., "MANIFEST instrument concept and related technologies," in Ground-based and Airborne Instrumentation for Astronomy IV, Proceedings of the SPIE 8446, 84467I (Sept. 2012).

[105] Lawrence, J. S., Brown, D. M., Brzeski, J., et al., "The MANIFEST fibre positioning system for the Giant Magellan Telescope," in Ground-based and Airborne Instrumentation for Astronomy V, Proceedings of the SPIE 9147, 914794 (Aug. 2014).

[106] Suyu, S. H., Treu, T., Blandford, R. D., et al., "The Hubble constant and new discoveries in cosmology," ArXiv e-prints (Feb. 2012).

[107] Riess, A. G., Macri, L. M., Hoffmann, S. L., et al., "A 2.4\% Determination of the Local Value of the Hubble Constant," ArXiv e-prints (Apr. 2016).

[108] Riess, A. G., Macri, L., Casertano, S., et al., "A 3\% Solution: Determination of the Hubble Constant with the Hubble Space Telescope and Wide Field Camera 3," Astrophysical Journal 730, 119 (Apr. 2011).

[109] Levi, M., Bebek, C., Beers, T., et al., "The DESI Experiment, a whitepaper for Snowmass 2013," ArXiv e-prints (Aug. 2013).

[110] DESI Team, "DESI Final Design Report Part I: Science, Targeting, and Survey Design," http://desi.lbl.gov/wp-content/uploads/2014/04/fdr-science-biblatex.pdf (2016).

[111] DESI Team, "DESI Final Design Report Part II: Instrument Design," http://desi.lbl.gov/wpcontent/uploads/2014/04/fdr-instrument-biblatex2.pdf (2016).

[112] Dalton, G., Trager, S., Abrams, D. C., et al., "Project overview and update on WEAVE: the next generation wide-field spectroscopy facility for the William Herschel Telescope," in Ground-based and Airborne Instrumentation for Astronomy V, Proceedings of the SPIE 9147, 91470L (July 2014).

[113] de Jong, R. S., Barden, S., Bellido-Tirado, O., et al., "4MOST: 4-metre Multi-Object Spectroscopic Telescope," in Ground-based and Airborne Instrumentation for Astronomy V, Proceedings of the SPIE 9147, 91470M (July 2014).

[114] Takada, M., Ellis, R. S., Chiba, M., et al., "Extragalactic science, cosmology, and Galactic archaeology with the Subaru Prime Focus Spectrograph," Publications of the Astronomical Society of Japan 66, R1 (Feb. 2014). 